\title{
MAPK signaling is necessary for neurogenesis in Nematostella vectensis
}

\author{
Michael J. Layden ${ }^{*}$, Hereroa Johnston ${ }^{3}$, Aldine R. Amiel ${ }^{3}$, Jamie Havrilak', Bailey Steinworth², Taylor Chock²,
} Eric Röttinger ${ }^{3}$ and Mark Q. Martindale $2^{2^{*}}$

\begin{abstract}
Background: The nerve net of Nematostella is generated using a conserved cascade of neurogenic transcription factors. For example, NvashA, a homolog of the achaete-scute family of basic helix-loop-helix transcription factors, is necessary and sufficient to specify a subset of embryonic neurons. However, positive regulators required for the expression of neurogenic transcription factors remain poorly understood.

Results: We show that treatment with the MEK/MAPK inhibitor U0126 severely reduces the expression of known neurogenic genes, Nvath-like, NvsoxB(2), and NvashA, and known markers of differentiated neurons, suggesting that MAPK signaling is necessary for neural development. Interestingly, ectopic NvashA fails to rescue the expression of neural markers in U0126-treated animals. Double fluorescence in situ hybridization and transgenic analysis confirmed that NvashA targets represent both unique and overlapping populations of neurons. Finally, we used a genome-wide microarray to identify additional patterning genes downstream of MAPK that might contribute to neurogenesis. We identified 18 likely neural transcription factors, and surprisingly identified $\sim 40$ signaling genes and transcription factors that are expressed in either the aboral domain or animal pole that gives rise to the endomesoderm at late blastula stages.
\end{abstract}

Conclusions: Together, our data suggest that MAPK is a key early regulator of neurogenesis, and that it is likely required at multiple steps. Initially, MAPK promotes neurogenesis by positively regulating expression of NvsoxB(2), Nvath-like, and NvashA. However, we also found that MAPK is necessary for the activity of the neurogenic transcription factor NvashA. Our forward molecular approach provided insight about the mechanisms of embryonic neurogenesis. For instance, NvashA suppression of Nvath-like suggests that inhibition of progenitor identity is an active process in newly born neurons, and we show that downstream targets of NvashA reflect multiple neural subtypes rather than a uniform neural fate. Lastly, analysis of the MAPK targets in the early embryo suggests that MAPK signaling is critical not only to neurogenesis, but also endomesoderm formation and aboral patterning.

\section{Background}

Cnidarians (e.g., corals, sea anemones, and "jellyfish") are the closest group of animals to the Bilateria (all bilaterally symmetrical animals such as vertebrates, flies, and nematodes), and are thus an important taxon to understand the origin and evolution of complex traits such as nervous systems [1]. Nematostella vectensis is a proven cnidarian model because it is easy to maintain in

\footnotetext{
* Correspondence: layden@lehigh.edu; mamartin@whitney.ufl.edu 'Department of Biological Sciences, Lehigh University, Bethlehem, PA, USA ${ }^{2}$ The Whitney Marine Laboratory for Marine Science, University of Florida, St. Augustine, Florida, USA

Full list of author information is available at the end of the article
}

laboratory culture, its genome is sequenced and annotated, and multiple tools exist for functional genetic approaches [1-6].

The Nematostella nervous system comprises endodermal and ectodermal nerve nets $[7,8]$. Neuronal cell bodies are arranged in a "salt-and-pepper pattern" such that individual neurons are scattered amongst non-neural cell types. Differentiating neurons are first detected in the late blastula stage before invagination of the presumptive endodermal plate [7,9]. Salt-and-pepper expression of both $N v \operatorname{soxB}(2)$ and Nvath-like (also called Nvarp3) are the earliest known neurally expressed genes and they define proliferative neural progenitor cells $[9,10]$. Shortly 
after Nvath-like and NvsoxB(2) are detected, expression of post mitotic neural markers such as NvashA and Nvelav1 is detected $[7-9,11]$. Nvelav1 is broadly expressed in a large number of neurons, though it is still unclear if it is a pan-neuronal marker in Nematostella [8]. Morpholino (MO)-mediated knockdown of either NvsoxB(2) or Nvathlike results in a loss of expression of both Nvelav1 and the neural subtype marker NvashA $[9,10]$. NvashA is expressed in a smaller number of developing neurons at embryonic stages [11]. Functional characterization of $\mathrm{Nva-}$ sh $A$ clearly demonstrated that it is necessary and sufficient to promote expression of the neural marker Nvelav1 and a number of putative neural subtype markers [11]. Based on previous work, a reasonable model for Nematostella neurogenesis is that Notch activity selects Nvath-like+ $N v \operatorname{sox} B(2)+$ neural progenitors from a pool of naïve cells; daughters of those progenitor cells express additional neurogenic genes such as NvashA, which in turn promote expression of post mitotic markers such as Nvelav1 and neural subtype markers [1]. However, the upstream inductive mechanisms responsible for initiating neurogenic cascades in Nematostella remain elusive, as do the molecular programs that give rise to NvashA-independent neural subtypes during neurogenesis.

FGF, Wnt, BMP, and Mitogen-activated protein kinase (MAPK) signaling cascades regulate neural induction in multiple species [12-17]. FGF, Wnt, and MAPK all promote neural development in other species $[12-14,16]$, whereas BMP activity is best known for its role in suppressing neural induction of the forming central nervous systems of model systems $[14,15,18]$. In Nematostella the role of these signaling pathways during neural induction is unclear. Disruption of Wnt signaling does result in neural phenotypes. However, the phenotypes are attributable to disrupted axial patterning [19-21]. Neural phenotypes resulting from loss and gain of BMP activity are complicated in that either manipulation results in loss of neurons, and neural phenotypes are restricted to larval stages [22, 23]. FGF-mediated MAPK signaling is one of many ways to initiate a receptor tyrosine kinase (RTK) cascade. Investigation of FGF signaling in Nematostella has primarily focused on its role in apical organ formation, and no broad neural phenotypes are reported, with the caveat that the current array of neural markers did not exist at the time of the initial study [24]. To date, the impact of MAPK signaling on neurogenesis has not been reported in Nematostella.

RTK signaling cascades are characterized by a series of kinases that are activated by upstream kinase and in turn activate a downstream kinase. Near the end of the cascade, activated MEK kinase phosphorylates ERK, which can translocate to the nucleus to phosphorylate and activate a number of transcription factors. Multiple RTK signaling cascades converge on MEK. For example, in
Nematostella, FGF signaling controls apical organ formation at larval stages, and pharmacological inhibition of MEK is able to phenocopy gene-specific MOmediated knockdown of the FGF-receptor $N v f g f R a$ [24]. The number of MAPK-like pathways that could be acting to regulate neural development in Nematostella is large. There are at least 12 FGF-like ligands and two FGF receptors in the Nematostella genome [2, 25]. Two ligands and one receptor (Nvfgfa1, Nvfgfa2, NvfgfRa) are expressed in the vegetal hemisphere/apical domain, the ligand Nvfgf8 is expressed in the animal hemisphere and its descendants, and the receptor $N v f g f R b$ in derivatives of both poles [24-26]. There are at least 25 additional receptors (Johnston \& Röttinger, unpublished) that could activate MEK/ERK signaling in Nematostella. Because the number of possible RTKs is relatively high, one strategy to better understand how these genes might be acting to regulate neurogenesis is to inhibit MEK. Thus, a number of possible signaling pathways can be simultaneously disrupted to determine if MAPK signaling contributes to neural development.

Here we use U0126, a potent and specific inhibitor of MEK [27], to test if MAPK signaling plays a role in neurogenesis, and to determine if we can use this disruption to identify other putative neural genes in the early embryo. We show that treatment with U0126 reduces expression of Nvath-like, NvsoxB(2), NvashA, and postmitotic neural markers. Loss of embryonic neurogenesis following U0126 treatment cannot be rescued by NvashA, suggesting U0126 treatment desensitizes embryonic cells from responding to proneural cues. We performed a genome-wide expression array to identify new MEK downstream targets, enabling us to characterize 18 novel salt-and-pepper-expressed genes. We tested the role of the putative FGF receptor NvfgfRa, which was the most likely candidate pathway disrupted by U0126 in regards to neural marker expression. However, we found no evidence suggesting that $N v f g f R a$ signaling regulates neurogenesis or any of the salt-and-pepper genes identified in the U0126 microarray. We also investigated the relationship of these novel salt-and-pepper genes with NvashAdependent neurogenesis. We confirmed one positive and one negative target of NvashA, Nvvsx-like and Nvath-like respectively. Lastly, we expanded our study to gain insight into whether $N v a s h A$ regulated one or multiple neuronal subtypes. Using transgenic animals and double fluorescent mRNA in situ hybridization, we confirmed that NvashA regulates at least two distinct neural subtypes; however, based on its expression pattern at later developmental stages [11], this number is likely much higher. Additionally, the identification of $\sim 80$ genes that are expressed in the presumptive endomesoderm and aboral pole suggests that MAPK signaling plays a key role in multiple aspects of Nematostella embryogenesis. Taken together, our data 
and previously published results allow us to incorporate MEK, a key regulator, in a preliminary gene regulatory network describing embryonic NvashA-dependent neurogenesis, and provided us with a list of additional likely neurogenic genes, aboral genes, and endomesodermal genes for future studies.

\section{Results}

\section{Determining ideal dose of U0126 to inhibit MEK inhibition}

To determine the most effective concentration of U0126 to use for our analyses, we treated fertilized zygotes with increasing concentrations of U0126 and analyzed expression of the previously described U0126 target Nvfgfa 1 as well as two markers of the animal hemisphere, Nvsprouty and Nvbra [24, 25, 28, 29] (Fig. 1). Nvsprouty was expressed throughout a central domain at the animal pole in the presumptive endoderm (Fig. 1a, whereas Nvbra was expressed in a central ring surrounding the future endodermal tissue (Fig. 1h). These two additional markers were chosen because we observed gastrulation failures in preliminary tests of the U0126 compound on early embryos and, in bilaterians, expression of sprouty homologs is downstream of FGF signaling (Fig. 2) [30]. U0126 treatments of $1-10 \mu \mathrm{M}$ reduced Nvsprouty expression, but had little to no impact on the expression of Nvfgfa 1 or Nvbra (Fig. 1a-d, g-j, m-p; Additional file 1:
Table S1). However, at a concentration of $15 \mu \mathrm{M}$, both Nvfgfa1 and Nvsprouty were undetectable in U0126treated animals (Fig. 1e, q; Additional file 1: Table S1). Interestingly, treatment with $15 \mu \mathrm{M}$ U0126 induced ectopic Nvbra expression within the endoderm-forming central domain (Fig. 1k, l), suggesting that MEK signaling actively represses Nvbra in the most central endodermforming domain. Based on the ectopic expression of Nvbra in the central domain as well as the complete inhibition of Nvsprouty and Nvfgfa1 expression in $15 \mu \mathrm{M}$ treatments (Fig. 1e, k, q; Additional file 1: Table S1), we concluded that $15 \mu \mathrm{M}$ of U0126 is an effective dose, and it is unlikely phenotypes are due to toxicity.

\section{Inhibition of MEK prevents ERK phosphorylation and gastrulation}

To ensure U0126 treatment is inhibiting MEK, we screened embryos treated with either dimethyl sulfoxide (DMSO) as a control or U0126 for the presence of phosphorylated ERK (pERK) (Fig. 2) [31]. pERK staining in controls was detected at low levels in most cells of the blastula, but was enriched in the presumptive endoderm cells that undergo invagination (Fig. 2a). In U0126 treatments, a positive pERK signal above the general ectodermal staining was not detected (Fig. 2f). We further analyzed the morphological phenotypes induced by the

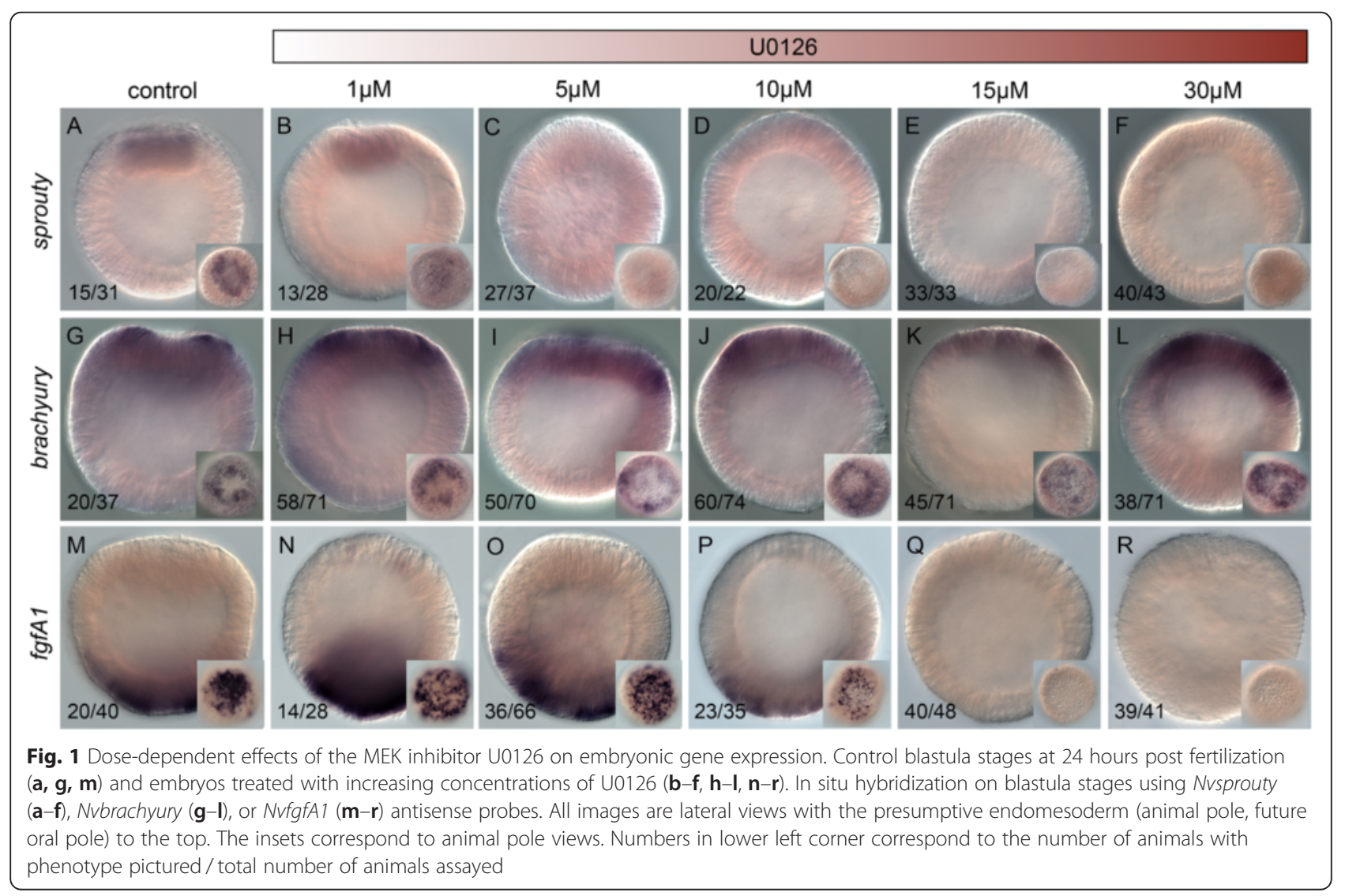




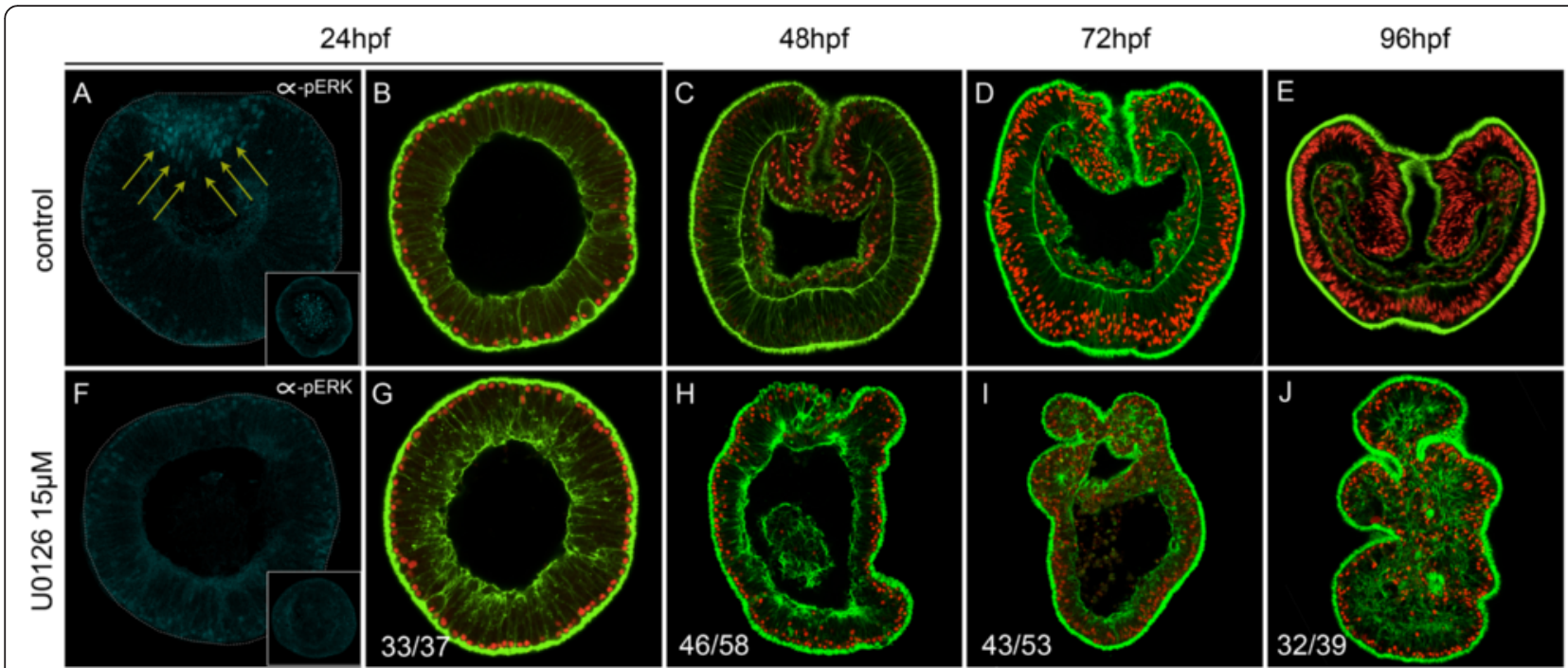

Fig. $\mathbf{2}$ U0126 blocks ERK activation, endomesoderm formation, and gastrulation. a-e Control embryos. $\mathbf{f}-\mathbf{j}$ U0126-treated embryos. a, f Confocal $z$-sections using anti-phospho-ERK (cyan) to visualize activated ERK. The gray dotted lines indicate the outline of the embryo and yellow arrows the accumulation of phosphorylated ERK (pERK)-positive cells above background levels. $\mathbf{b}-\mathbf{e}, \mathbf{g}$-j Confocal z-sections using phalloidin (green) to show f-actin filaments and propidium iodide (red) to visualize the nuclei. $\mathbf{a}, \mathbf{b}, \mathbf{f}, \mathbf{g}$ Blastula stages [24 hours post fertilization (hpf)]. $\mathbf{c}, \mathbf{h}$ Late gastrula stages (48 hpf). d, i Early planula (72 hpf). e, $\mathbf{j}$ Late planula (96 hpf). All images are lateral views with the animal/oral pole to the top. The insets in $\mathbf{a}$ and $\mathbf{f}$ correspond to animal pole views. Ratios in $\mathbf{g}$-j indicate the number of embryos displaying the phenotype shown in the image to the total number of analyzed embryos

disruption of ERK signaling and observed that treated embryos dramatically failed to gastrulate and form a gut (Fig. 2g-j) compared to control embryos (Fig. 2b-e).

\section{NvashA, Nvath-like, and NvsoxB(2) are globally downregulated in U0126-treated embryos}

We next tested if U0126 treatment would disrupt expression of the known neural genes. We treated embryos with U0126 and scored for expression of known neural genes NvashA, Nvath-like, and NvsoxB(2) (Fig. 3). mRNA in situ hybridization on U0126-treated and control animals during early gastrula stages [24 hours post fertilization (hpf) at $17^{\circ} \mathrm{C}$ ] (Fig. 3) revealed that all three genes-NvashA, NvsoxB(2), and Nvath-like-were globally reduced in U0126-treated animals (Fig. 3). However, they did not all display the same sensitivity to U0126. Nvath-like expression was undetectable in $82 \%$ and NvashA expression was undetectable in $96 \%$ of U0126-treated embryos (Fig. 3A, C). NvsoxB2 expression was dramatically reduced, both in terms of the number of cells expressing it and in the level of expression (Fig. 3B), but expression was detectable in many more embryos than observed for either NvashA or Nvath-like. Interestingly, we identified $N v \operatorname{sox} B(2)$ as being maternally expressed using the SeaBase database of transcriptomes (Additional file 2: Figure S1) [32-34]. To address whether or not U0126 might have a more severe impact on $N v \operatorname{sox} B(2)$, we treated animals at late gastrula stages for 24 hours with U0126 or DMSO control. We observed that $N v \operatorname{sox} B(2)$ expression was not detectable in $87 \%$ of U0126-treated cells (Additional file 3: Figure S2A). We also observed a strong reduction in NvashA expression in animals treated with U0126 for 24 hours after completion of gastrulation (Additional file 3: Figure S2B). These data suggest that the earliest known neurogenic transcription factors are globally reduced in U0126-treated animals, which indicates that neurogenesis is disrupted by U0126.

\section{NvashA misexpression fails to rescue neurogenesis in U0126-treated animals}

To test if U0126 treatment impacted neurogenesis, treated animals were allowed to develop until a time equivalent to the late gastrula stage and screened for expression of the broadly expressed neural marker Nvelav1 and previously identified NvashA neural target genes by mRNA in situ hybridization. U0126 treatment dramatically reduced expression of neural markers, such that they were essentially undetectable (Fig. 4). Two exceptions were NvLWamide-like (PrtID\# 242283, http://genome.jgi.doe.gov/Nemve1/Nemve1.home.html) and NvanthoRFamide, which showed staining in a few cells (Fig. 4A, 4H'), but both were reduced compared to control DMSO-treated animals. We conclude that treatment with U0126 results in a nearly complete loss of embryonic neural marker expression.

We next aimed to determine if loss of neural markers in U0126-treated animals could be rescued by misexpression of neurogenic transcription factors. $N v s o x B(2)$ and Nvath- 


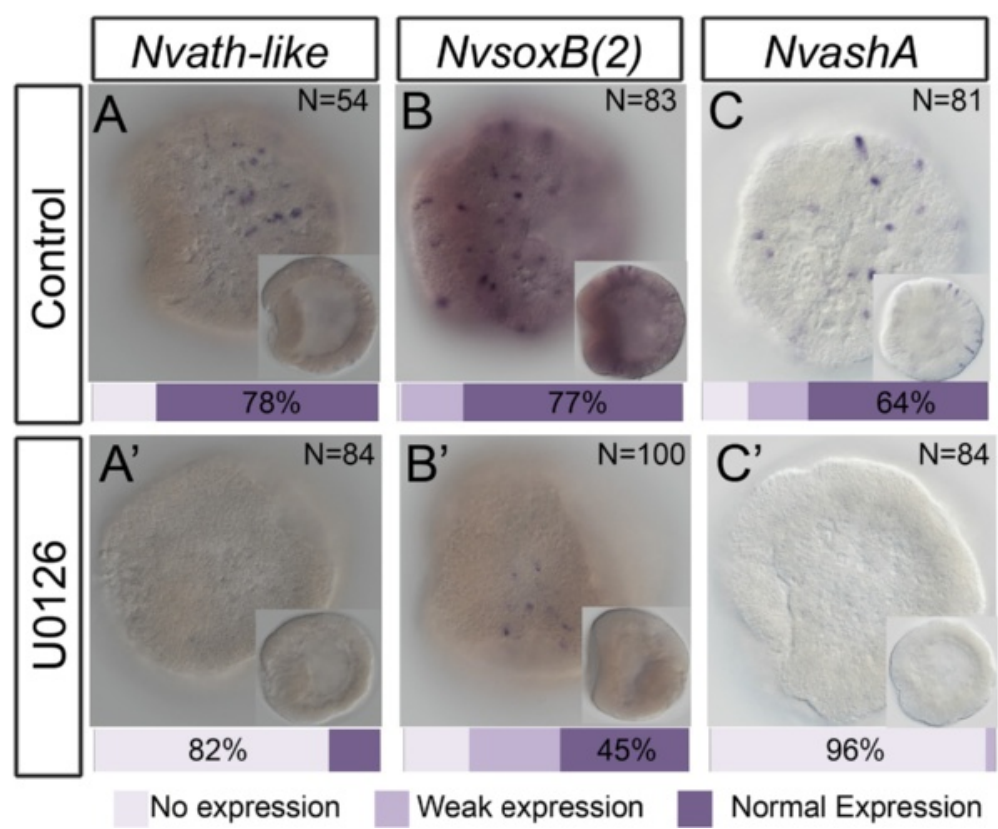

Fig. 3 U0126 treatment results in a global decrease in neurogenic transcription factors. mRNA in situ expression of Nvath-like (A), NvsoxB(2) (B), and NvashA (C) in control embryos treated with in 1/3x artificial seawater (ASW) with $0.1 \%$ dimethyl sulfoxide (DMSO). Expression of Nvath-like $\left(\mathbf{A}^{\prime}\right), \operatorname{NvsoxB}(2)\left(\mathbf{B}^{\prime}\right)$, and NvashA $\left(\mathbf{C}^{\prime}\right)$ in animals treated with $15 \mu \mathrm{m}$ U0126 in 1/3x ASW with $0.1 \%$ DMSO. Embryos were classified and quantified as the percent having normal expression, weak expression, or no expression. Refer to key in figure for classification of phenotypes. The phenotypic class with the highest percentage of embryos is indicated. All embryo images are of early gastrula stage. The main figure panels are ectodermal focal planes of lateral views with the presumptive oral side to the left. The insets show deeper focal planes used to confirm embryonic stage

like act upstream of NvashA, both have much broader roles than NvashA in neurogenesis, and both are expressed in neural progenitor cells $[9,10]$. However, misexpression phenotypes are not reported for either of these genes. We were also concerned that over-activation of progenitor cell markers could result in a loss of neural markers due to an inability to transition from an undifferentiated to a differentiated state [35]. Thus, observing no rescue with misexpression of either $N v \operatorname{soxB}(2)$ or Nvathlike could represent a false negative neural phenotype. Nvash $A$ is expressed in differentiated cells, and misexpression of NvashA has already been shown to be sufficient to induce ectopic neural marker expression $[9,11]$. We injected in vitro transcribed NvashA:venus mRNA and treated animals with U0126. Surprisingly, we observed no rescue of the neural markers (Fig. $4 \mathrm{~A}^{\prime \prime}-\mathrm{H}^{\prime \prime}$ ). To ensure that NvashA:venus behaves as previously reported $[6,11]$, we treated animals injected with NvashA:venus mRNA with U0126. High levels of NvAshA:Venus were still detectable and localized to the nucleus (Additional file 4: Figure S3). We conclude that NvashA is not sufficient to rescue neural marker expression in U0126-treated animals.

The failure of NvashA to rescue U0126-induced loss of neurogenesis might be a consequence of the reduced NvsoxB(2) and Nvath-like expression in U0126-treated animals. To address this hypotheses we took advantage of the previous observation that NvashA restores neural marker expression lost in animals with hyperactivated Notch signaling even though NvsoxB(2) and Nvath-like remain strongly downregulated [36]. To compare the downregulation of NvsoxB(2) and Nvath-like induced by hyperactivation of Notch to the downregulation observed in U0126-treated animals, we performed mRNA in situ hybridization experiments in NvnotchICD:venusinjected animals (Additional file 3: Figure S2). Injection of NvnotchICD:venus mRNA resulted in $83 \%$ of animals showing no or weak $N v \operatorname{sox} B(2)$ expression and $88 \%$ of animals showing no Nvath-like expression (Additional file 3: Figure $\mathrm{S} 2$ ). Both genes were more severely reduced in NvnotchICD:venus-injected animals than they had been in U0126-treated animals (compare Fig. 3A, B to Additional file 3: Figure S2A, B). Taken together with previous reports that NvashA rescues neuronal loss induced by Notch hyperactivity without increasing $N v \operatorname{soxB}(2)$ or Nvath-like expression, we argue that U0126 disrupts neurogenesis in two ways. It inhibits the expression of the neurogenic transcription factors Nvath-like, $N v \operatorname{soxB}(2)$, and NvashA, and it disrupts a yet unknown pathway that is also required for NvashA to promote neural fates. 


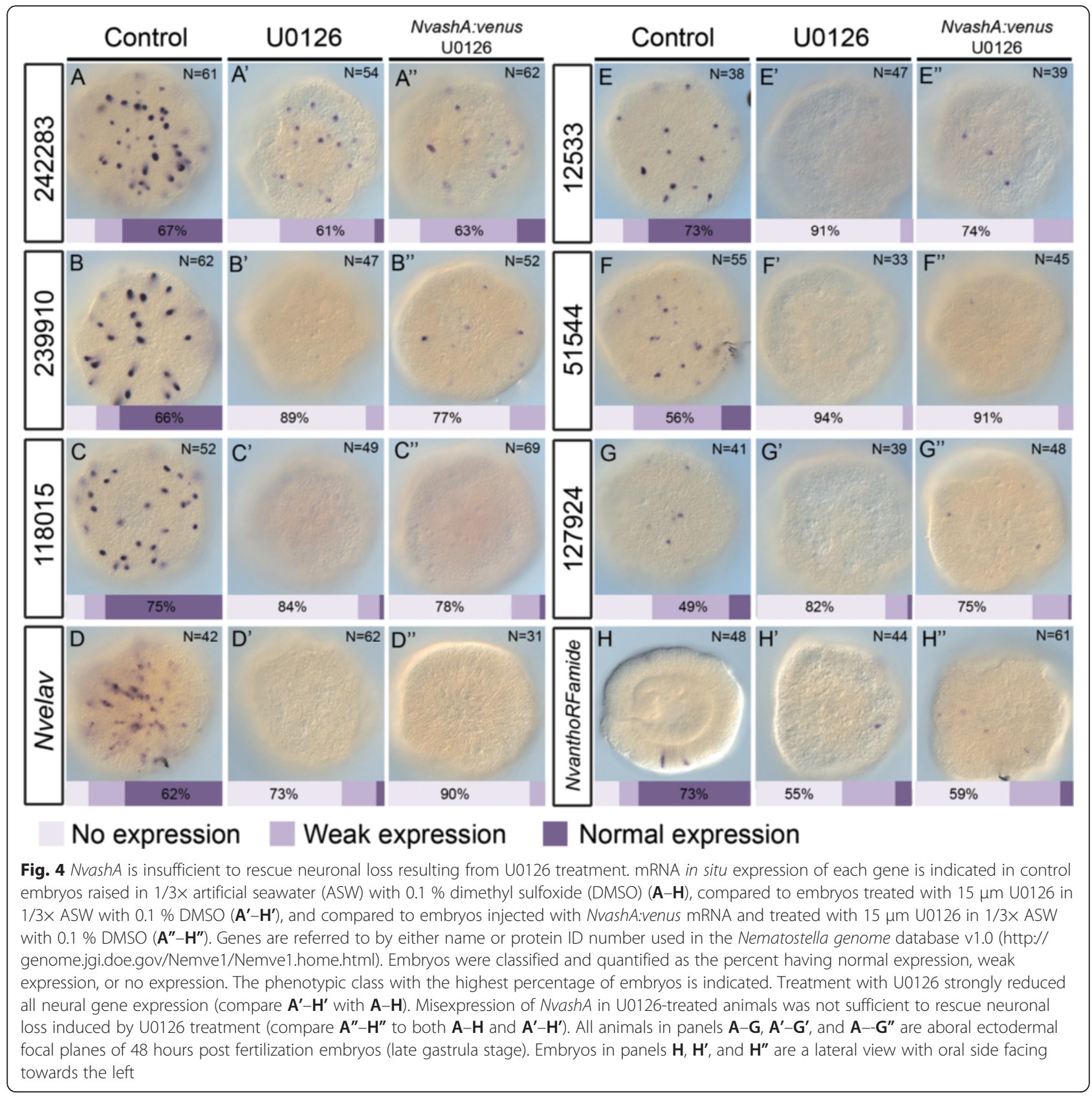

\section{NvashA target genes likely represent multiple distinct neuronal subtypes}

We wanted to confirm that NvashA regulates multiple neuronal subtypes. Although NvashA is expressed in a subset of the nervous system, subtle differences in the expression domains of NvashA targets suggests that they describe distinct neural subtypes [11]. To test if NvashA regulates distinct neural subtypes we performed double fluorescent in situ hybridizations and created stable meganuclease-mediated transgenic reporters for two of the NvashA targets using an approximately 2000-basepair genomic region immediately upstream of the start codon for both genes to drive expression of mCherry fluorescent protein (Fig. 5) [37]. We chose NvLWamidelike (PrtID\# 242283) (Fig. 5a, b) and Nvserum amyloid A-like (PrtID\# 239910) (Fig. 5a, c) because these genes represent NvashA targets with overlapping but slightly different expression patterns during development, and both genes were strongly downregulated in the U0126 microarray (see below; Additional file 5: Table S2, Additional file 6: Table S3). Both genes were expressed broadly throughout the aboral region of the embryo, but the NvLWamide-like expression domain extended more orally and encompassed a larger domain than that of 

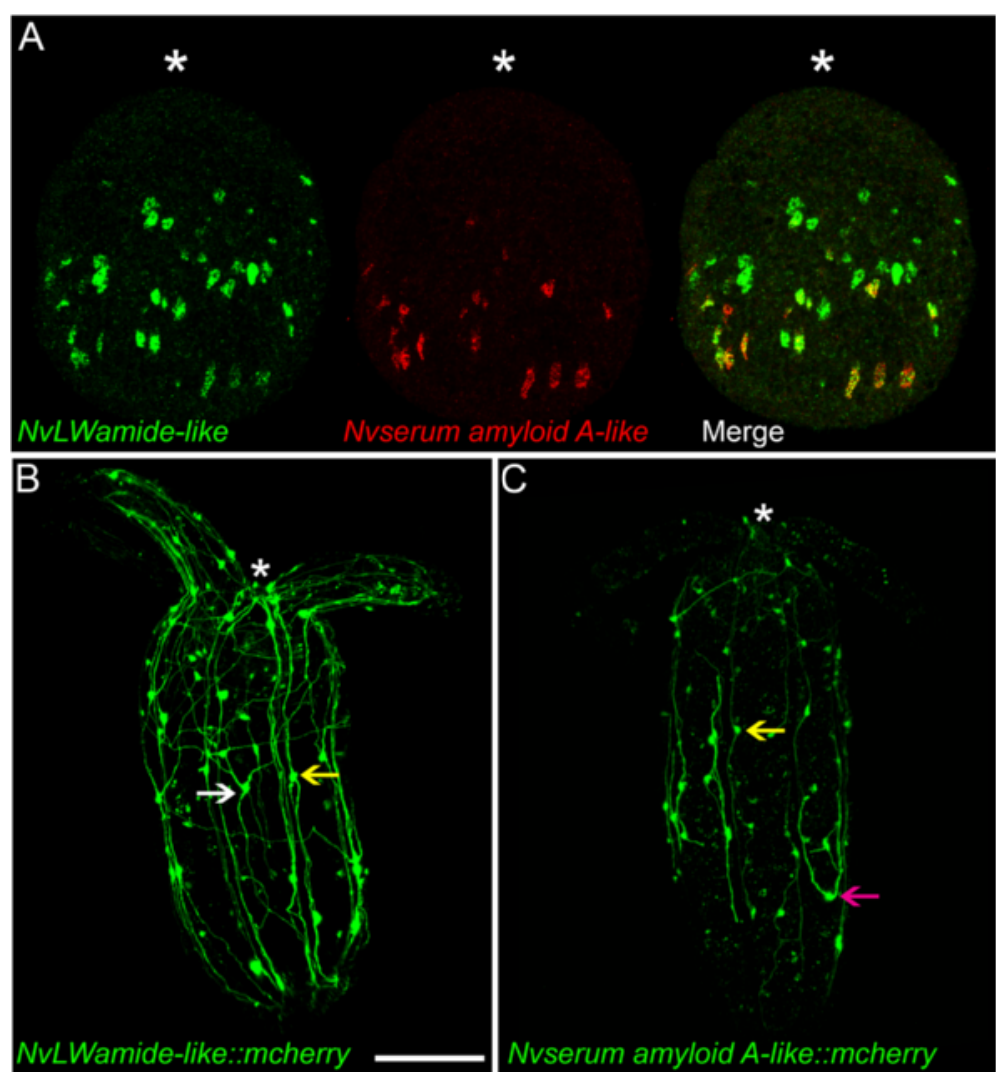

Fig. 5 NvashA regulates multiple neuronal subtypes. Shown are three-dimensional projections of two juvenile polyps shown with the oral side towards the top of the image. a Double fluorescent in situ hybridization of NvLWamide-like (red) and Nvserum amyloid A-like (green) in a late gastrula stage embryo. $\mathbf{b}$ The transgenic line for NvLWamide::mcherry expression is shown. Neural soma and neurites are observed throughout the body column and tentacles. Ectodermal neurons with three projections are observed in the body column (white arrow), which are not found in the other transgenic line. c The transgenic line for Nvserum amyloid A-like::mcherry is shown. There are many fewer neurons compared to NVLWamide::mcherry, but characteristic neurons are present. The U-shaped neuron that has two orally projecting neurites (pink arrow) is specific to this transgenic line. Both lines have neurons that are located just over the mesenteries and send projections orally and aborally in neural tracts overlaying the mesenteries (yellow arrows). In all images, asterisks indicate relative position of mouth

Nvserum amyloid A-like (Fig. 5a) [11]. NvLWamide-like was expressed in more cells than Nvserum amyloid A-like (Fig. 5a). Double fluorescent mRNA in situ hybridizations revealed that many of the Nvserum amyloid A-like expressing neurons (Fig. 5a, red) were also positive for NvLWamide-like (Fig. 5a, green). However, there were many examples of NvLWamide-like-only-positive cells and few examples of Nvserum amyloid A-like-only-positive cells (Fig. 5a). Thus, it is likely that there are at least three molecularly distinct neural subtypes that require NvashA for development.

We next compared the neurons labeled in each transgene to determine if the neurite projections from Nvserum amyloid A-like and NvLWamide-like neurons are similar or distinct. Although there were a number of neurons with similar neurites that projected along the oral-aboral axis in each transgene (Fig. 5b, c, yellow arrows), each transgene also labeled neurons with distinct morphologies (Fig. 5b, c, white and pink arrows, respectively). Consistent with the mRNA in situ hybridization results, the NvLWamide-like::mCherry labeled more cells than NvSerum Amyloid Alike::mCherry (compare Fig. 5b and c). Interestingly, there were distinct cell types present in each line [for example, NvLWamide-like::mCherry was expressed in ectodermal cells that had three neurites extending from the soma (Fig. 5b, white arrow), and NvSerum Amyloid A-like had unique neurons with neurites that extended out of either side of the soma and projected orally to form U-shaped neurons (Fig. 5c, pink arrow)] that could be reproducibly identified in individual transgenic animals. Although more extensive characterization is required for each of these transgenic lines, the fact that Nvash $A$ targets have distinct expression patterns and that neurons described by the transgenes of two $\mathrm{Nva}$ shA targets display distinct morphologies support the conclusion that NvashA regulates multiple neuronal subtypes. 
Identification of 100 genes downregulated and 22 genes upregulated by U0126 treatments

To determine if U0126 regulates other potential neurogenic genes and to identify novel targets of MEK signaling, we applied a forward molecular approach using a genome-wide expression microarray. Zygotes were treated with U0126 or DMSO until late blastula stage. At that point mRNA was extracted and used to generate labeled cDNA, which was hybridized to a custom expression microarray (Nimblegen, Inc.) that represents 24,021 predicted $N$. vectensis gene models [28]. The Pearson's correlation factor between biological replicates was mediocre (0.69), however, 926 genes were significantly $(P<0.05)$ upregulated and 1176 genes were significantly $(P<0.05)$ downregulated in U0126-treated embryos (Additional file 6: Table S3). Because our focus was to identify early embryonic patterning events, we screened the lists of genes to identify transcription factors, signaling molecules, and signaling modulators. This resulted in the identification of 100 genes that were downregulated and 22 genes that were upregulated by U0126 (Additional file 7: Table S4; Additional file 8: Table S5 [2, 7, 9, 11, 19, 24, 26, 28, 38-57]). Out of the 122 selected genes, the vast majority $(86 / 122)$ possess strong orthology with members of various families of transcription factors (i.e., forkhead, pointed, homeodomain). Twenty-seven genes potentially encode ligands, modulators, or receptors of signaling pathways (i.e., Wnts), and nine genes show similarities with adhesion molecules, metalloproteases, or RNA binding proteins with described developmental functions in bilaterians (i.e., Ncam, Tolloid, Vasa) (Additional file 6: Table S3). To distinguish between previously published genes and newly identified ones, we used the best Blast Hit identification followed by "-like". Thus, with the exception of Nvhes-like and Nvath-like [9, 51], all gene names containing "-like" designate gene products described for the first time in this study.

\section{Identification of putative neural genes}

Neural genes are predicted to have a salt-and-pepper expression pattern, and thus determining the expression pattern of the 122 target genes would identify which genes exhibit salt-and-pepper expression associated with neural genes. We performed whole mount in situ hybridizations in the developing embryo at blastula stages (24 hpf) and at the end of gastrulation (48 hpf, Fig. 6). We excluded 16 genes because their expression patterns are already described at the blastula stage, but we did include genes whose early expression patterns had not yet been described (Nvgata, for example [23]). We were able to obtain clones for 98 of the remaining 106 genes and synthesized anti-sense probes. All original publications corresponding to a given gene (sequence identification and/or gene expression pattern) can be found in Additional file 7: Table S4 and Additional file 8: Table S5. Our in situ screen revealed reproducible patterns for 60 of the 98 genes we screened. Patterns could be grouped into one of three categories: (1) genes expressed in the animal hemisphere/presumptive endomesoderm of the blastula (24/98) (Additional file 9: Figure S4); (2) genes expressed in the vegetal hemisphere/aboral domain of the blastula (18/98) (Additional file 9: Figure S4); and (3) genes with the characteristic salt-and-pepper pattern consistent with being neural genes (18/98) (Fig. 6). We found no link between genes that are expressed broadly in the animal and vegetal hemispheres and neurogenesis, and thus will not discuss these genes further here.

Genes expressed in individual cells throughout the ectoderm The 18 genes expressed in individual cells of the presumptive ectoderm at the blastula stage or in ectodermal body wall at the end of gastrulation were Nvsox2, Nvpea-like3, Nvcoup-like1 (also called NR-like 12), Nvcoup-like2 (also called NR-like 13) [55], Nvelav-like, NvemxLX, Nvhd145, Nvtailless-like, Nvvsx-like, Nvgfilike, Nvsox10-like, Nvhes-like3, Nvfoxq2-like3, NvfoxD3like, Nvdkk-like3, Nvvegef-like1, Nvhox2, and Nvhd052 (Fig. 6). Eight genes (Nvelav-like, NvemxLX, Nvtaillesslike, Nvgfi-like, Nvdkk3-like3, Nvvegf-like1, and Nvhox 2 and Nvhd052 we did not detect localized expression prior to the onset of gastrulation (Fig. 6E,F,N,P,ZaZd). Six genes (Nvpea3-like, Nvcoup-like1, Nvvsx-like, Nvsox10-like, Nvhes-like3, and Nvfoxq2-like3) were expressed in 5-15 cells (Fig. 6B, C, O, Q, R, Y). For four genes (Nvsox2, Nvcoup-like2, Nvhd145, and NvfoxD3-like), more than 15 cells were stained throughout the presumptive ectoderm at the blastula stage (Fig. 6A, D, M, Z).

At the end of gastrulation, we observed gene expression for all above-mentioned genes in individual cells (Fig. 6G-L, S-X, Ze-Zj, Zl). However, the localization and number of stained cells varied considerably. The only gene that appeared to be expressed in individual cells throughout the entire ectoderm (pharyngeal ectoderm, oral ectoderm, body wall ectoderm, sub-apical pole, and apical pole) was Nvhes-like3 (Fig. 6X), which is reminiscent of a previously reported gene, Nvash $A$ [11, 51]. Nvsox2 and Nvcoup-like1 (Fig. 6G, I) were also expressed in a salt-and-pepper manner throughout all ectodermal domains, except the pharyngeal ectoderm. The largest group of genes (Nvcoup-like2, Nvelav-like, NvemxLX, Nvhd145, Nvtailless-like, and Nvvsx-like) was detected in the body wall ectodermal as well as the sub-apical and apical domains (Fig. 6J, K, L, S, T, U). Cells expressing NvfoxD3-like and Nvdkk3-like3 (Fig. 6Zf, Zg) were detected in the oral and body wall ectoderm as well as the sub-apical pole domains; however, Nvsox10-like, Nvfoxq2-like3, and 

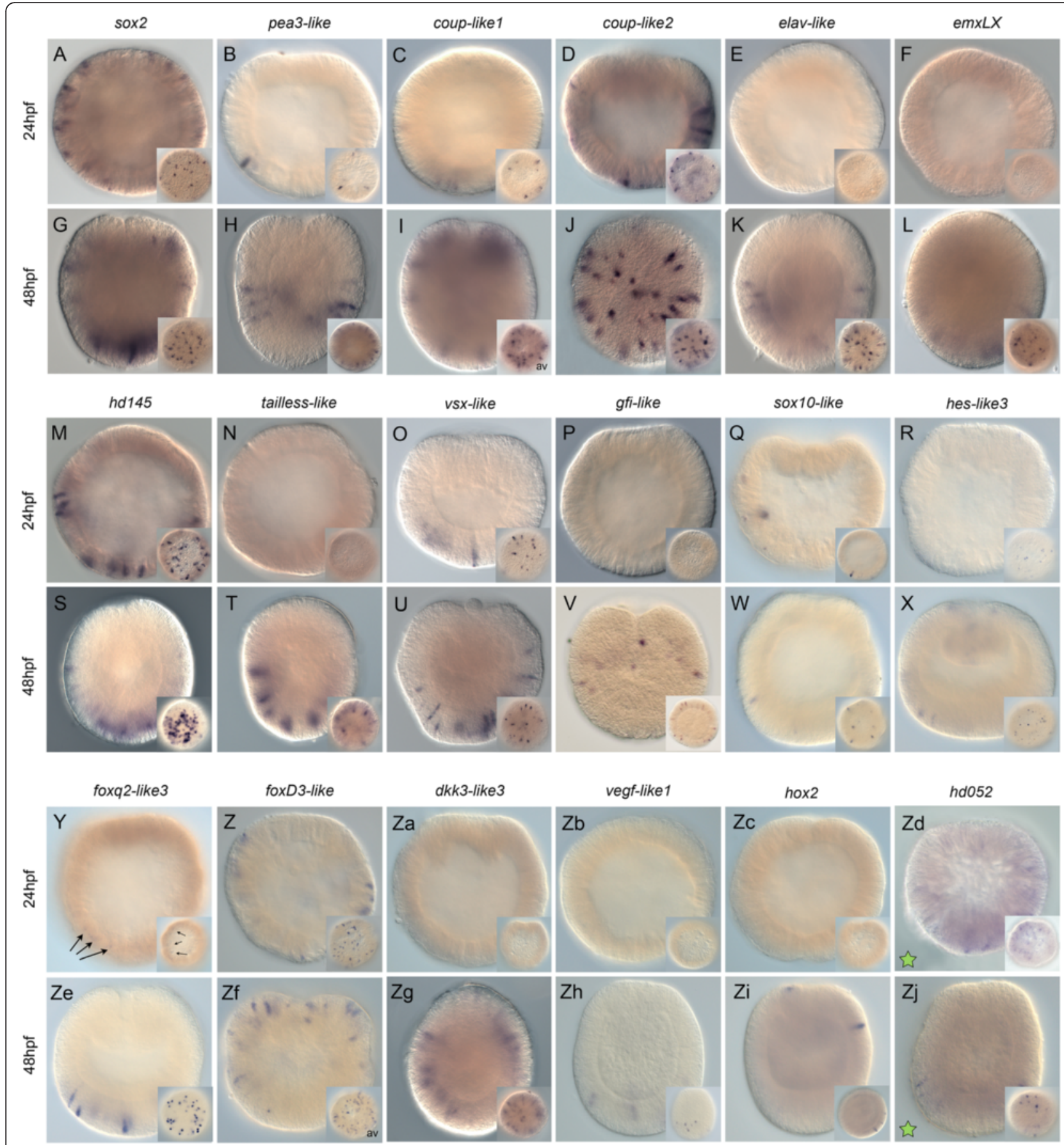

Fig. 6 U0126 targets expressed in individual cells throughout the ectoderm. Wild type salt-and-pepper gene expression analysis by in situ hybridization of genes differentially regulated by $\cup 0126$ treatments. All animals are either blastula [24 hours post fertilization ( $h p f)-\mathbf{A}-\mathbf{F}, \mathbf{M}-\mathbf{R}, \mathbf{Y}-\mathbf{Z d}$ ] or gastrula (48 hpf - G-L, $\mathbf{S}-\mathbf{X}, \mathbf{Z e}-\mathbf{Z j}$ ) stages. All images are lateral views with the animal pole to the top. The insets correspond to surface views. Antisense probes used as indicated. Green stars in Zd and Zj indicate that this gene was upregulated under U0126 conditions. All other genes were downregulated

Nvvegf-like were only detected in the body wall ectoderm and sup-apical domain (Fig. 6W, Ze, Zh). The genes with the most restricted expression domain were Nvpea3-like, Nvgfi-like, and Nvhox2, which were expressed in individual cells either within a circumferential territory (Fig. $6 \mathrm{H}, \mathrm{V}$ ) or in a patch within the body wall ectoderm (Fig. 6Zi), respectively. The variable expression patterns suggest that many of these genes are putatively expressed in distinct subsets of neurons, while a few broadly expressed genes might play larger roles during neural development. 
Temporal gene expression analysis of salt-and-pepper genes In situ hybridization provides crucial spatial information about gene expression but cannot be used quantitatively to determine the presence of maternal transcripts or zygotic upregulation of a given gene. However, this information is crucial for the design of functional studies, to predict potential genetic interactions, and to build gene regulatory networks [28]. In order to determine the temporal deployment of putative neural genes during early embryogenesis, we performed fine-scale quantitative reverse transcription PCR (RT-qPCR) on RNA/ CDNA that was sampled from unfertilized eggs and every $2-4 \mathrm{~h}$ during embryogenesis up to the late gastrula stage (48 hpf) Additional file 10: Figure S5. We analyzed 21 salt-and-pepper genes identified both in this and in previous studies, including the known neural genes such as Nvath-like and NvashA [9, 11]. Five salt-and-pepper genes (Nvfoxq2-like3, Nvcoup-like2, Nvelav-like, NvpaxA, and Nvvegf-like1) were detectable in zygotes, indicating that they are maternal genes (Additional file 11: Figure S6). However, it is not yet clear what the significance of these genes is because none of these genes have broad expression patterns that might indicate a key early role for them in neurogenesis. The majority of salt-andpepper genes, including the known neural regulators Nvath-like and NvashA, are not components of the maternally contributed mRNAs, which suggests that the earliest neural fates are induced in the embryo. As a group, the salt-and-pepper genes display a similar temporal deployment. The earliest zygotically regulated genes are Nvath-like (8-10 hpf), followed by Nvhes3 (10-12 hpf), NvfoxD3, Nvsox10-like (12-14 hpf), Nvfoxq2-like3, and Nvsox2 (16-18 hpf), and then the bulk of genes $(12 / 21)$ are upregulated at either 18-20 hpf (Nvcoup-like1, Nvcoup-like2, Nvgfi-like, Nvhd145, Nvtailless-like, Nvvsx-like, and Nvelav-like) or 20-24 hpf (Nvdkk-like3, Nvpea3-like, NvpaxA, Nvgcm, and Nva$\operatorname{sh} A$ ). Our temporal analysis demonstrated two key aspects. Nvath-like, which is the first neural gene to respond to treatment with DAPT and is thought to be the earliest acting neural gene [9], is in fact upregulated prior to other known neural genes. Second, the salt-andpepper genes all showed strong upregulation between 10 and $24 \mathrm{hpf}$. The upregulation of genes is continuous in that different genes are upregulated at different times throughout the 10-24 hpf window. This argues that once salt-and-pepper gene expression is initiated there is a steady increase in the expression of distinct saltand-pepper genes. Additionally, temporal differences in expression might reflect a hierarchal organization for their functions. The later concept is supported by the observation that Nvath-like (an upstream neural regulator) is detected much earlier than NvashA, which is expressed in differentiated neurons [9].

\section{NvFGFRa does not regulate salt-and-pepper gene expression} in the embryo

ERK-mediated FGF signaling can be inhibited by treatment with U0126 in Nematostella [24], and the NvfgfRa receptor is expressed broadly in the embryonic ectoderm, which makes it a likely candidate responsible for the U0126 neural phenotype and/or responsible for regulating some of the newly identified salt-and-pepper genes. We tested if $N v f g f R a$ might have an early neural phenotype by injecting the previously described $N v f g f R a$ $\mathrm{MO}$ into embryos [24] and scoring for changes in Nvath-like, NvashA, and the other 19 genes with saltand-pepper gene expression identified in the U0126 microarray. qPCR analysis comparing control $\mathrm{MO}$ and $N v f g r R a$ MO revealed that none of the salt-and-pepper genes responded to changes in $N v f g f R a$ levels (Fig. 7). These data suggest NvFgfra signaling is not the U0126 target responsible for the loss of salt-and-pepper expressed genes in the early gastrula. However, there is a maternal contribution of Nvfgfra signaling genes [24]. To confirm that this FGF signaling was unlikely the source of the neural phenotype, we allowed morphant animals to grow to $48 \mathrm{hpf}$ at $17{ }^{\circ} \mathrm{C}$. In parallel, we allowed wild-type animals to grow until $24 \mathrm{hpf}$, when they are approximately at the late blastula/early gastrula stage. Animals were then treated with U0126 from 24 to $48 \mathrm{hpf}$. This treatment regimen allows for the maternal action of FGF components, thus mimicking a potential flaw in the Nvfgfra morphant approach. qPCR analysis of NvashA and Nvfgfra (a target of Nvfgfra MO [24]) demonstrated that Nvfgfra MO effectively inhibited known targets, but not NvashA (Additional file 12: Figure S7B). We saw similar results with SU5402 treatment (Additional file 12: Figure S7B). However, U0126 treatment resulted in a strong reduction in all genes assayed (Additional file 12: Figure S7). These data argue that FGF signaling is not the source of the neural phenotype. Thus, although the neural phenotype observed in U0126-treated animals is robust, it is still unclear what signaling pathway(s) targeted by U0126 regulates salt-and-pepper gene expression in the embryonic ectoderm.

\section{NvashA regulates a small subset of the salt-and-pepper genes}

We next wondered if $N v a s h A$ acted to regulate any of the U0126 target genes. Based on the role of proneural genes in other animals we hypothesized that NvashA might act upstream of or suppress expression of subsets of the salt-and-pepper expressed U0126 targets. NvashA was knocked down or overexpressed using the previously described NvashA translation blocking $\mathrm{MO}$ and in vitro synthesized NvashA:venus mRNA [11]. We assayed U0126 salt-and-pepper target expression by qPCR after 


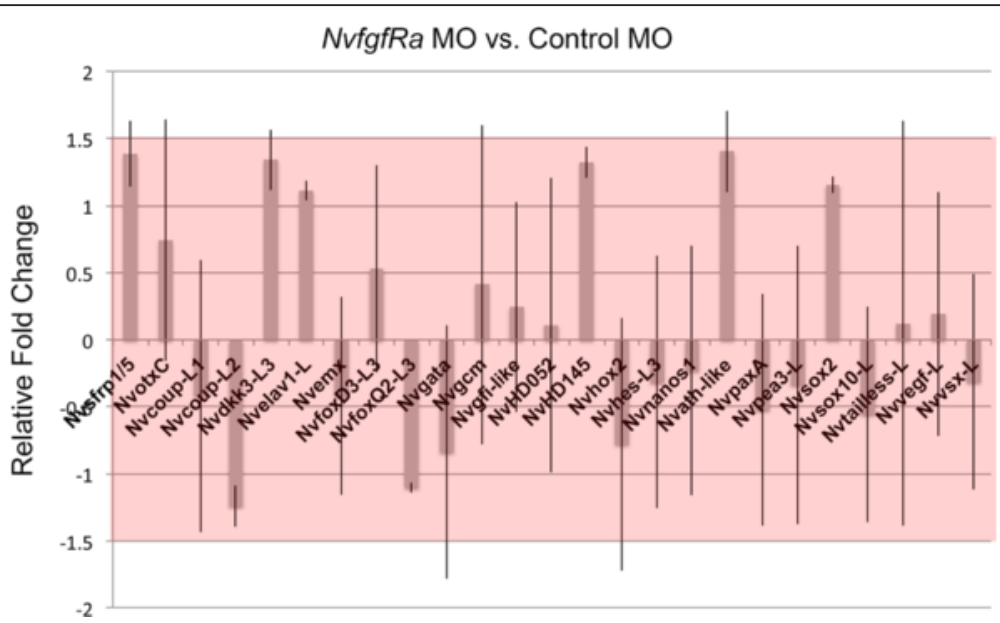

Fig. 7 NvfgfRa does not regulate U0126-dependent salt-and-pepper gene expression. Relative fold change calculated from quantitative polymerase chain reaction analysis of triplicate injections of the NvfgfRa morpholino $(M O)$ or a control MO is graphed. Broadly expressed genes, Nvsfrp1/5, NvotxC, and NvfgfRa, are included as controls. The remaining genes are the salt-and-pepper expressed genes identified by the U0126 microarray. The red box indicates a region $(1.5 \times$ to $-1.5 \times$ fold change) that was defined as the cut off for a significant change in expression. Error bars represent standard error

disruption of NvashA and confirmed any genes that showed a response to NvashA disruption by mRNA in situ hybridization (Fig. 8). We included six control genes. Four negative controls (Nvfgfra, Nvsfrp1/5-like, Nvsix3/6, and $N \operatorname{vot} x C$ ) represented broadly expressed regional patterning genes unlikely to respond to changes in Nvash $A$ function $[19,24,54]$. Two positive control genes, $N v L W a-$ mide-like (PrtID\# 242283) and canalicular multispecific anion transporter (PrtID\# 12533), are both confirmed positive targets of NvashA [11] and were both also downregulated in the U0126 array (Additional file 5: Table S2).

We first assayed for positive targets of NvashA. Overexpression of $N v a s h A$ increased the expression of $N v v s x-$ like, Nvgfi-like, Nvcoup-like1, Nvsox2, and Nvtailless-like (Fig. 8a, light gray bars). However, Nvvsx-like expression was weakly upregulated following injection of NvashA:venus mRNA (Fig. 8a light grey bars; Fig. 8d), but $N v v s x-$ like was the only upregulated gene that decreased after NvashA knockdown by $\mathrm{MO}$ injection (Fig. 8a, dark gray bars; Fig. 8b). These data argue that NvashA is necessary and sufficient for the expression of Nvvsx-like (Fig. 8a-d). NvashA was sufficient but not necessary to regulate Nvtailless-like in the early gastrula. Overexpression of NvashA increased the expression of Nvtailless-like (Fig. 8a, light gray bars). However, Nvtailless expression was not dependent on NvashA, as its expression level was not decreased in NvashA morphants (Fig. 8a, dark gray bars). Nvgfi-like, Nvcoup-like1, and Nvsox 2 also all showed increases in expression following injection of NvashA mRNA (Fig. 8a, light gray bars). However, injection of NvashA MO also resulted in increased expression of all three genes (Fig. 8a, dark gray bars). Because reciprocal phenotypes are not observed between mRNA-injected and MO- injected animals, and both morphant and overexpression phenotypes were similar, it is unclear if changes in expression of Nvcoup-like1, Nvsox2, and Nvgfi-like reflect normal Nvash $A$ activity. Thus, we exclude these genes as targets of NvashA until NvashA mutant analysis can confirm that they are downstream of NvashA in the early gastrula. We conclude that Nvvsx-like is a positive target of NvashA.

We next investigated the 10 genes, Nvath-like, NvemxLx, Nvhes-like3, Nvsox10-like, NvpaxA, Nvgcm, Nvgfi-like, Nvhd145, Nvcoup-like2, and Nvelav1, that displayed changes in expression consistent with being putative negative targets of NvashA. Nvhd145 and Nvcouplike 2 expression was reduced in NvashA-overexpressing animals, but no change in either gene was observed in NvashA morphants (Fig. 8a; Additional file 4: Figure S3). These data suggest that although NvashA is sufficient to suppress Nvhd145 and Nvcoup-like2, it does not likely regulate these genes during embryonic stages. Nvathlike, NvemxLx, Nvhes-like3, Nvsox10-like, NvpaxA, Nvgcm, Nvgfi-like, and Nvelav1 showed increased expression at early gastrula stages in NvashA morphant animals (Fig. 8a, light gray bars), which suggests that they might be negative targets of NvashA. Only Nvathlike and NvemxLx showed reciprocal changes in expression in NvashA gain and loss of function (Fig. 8a, dark gray bars versus light gray bars). We were only able to confirm the changes in Nvath-like expression by mRNA in situ hybridization (Fig. 8e-g). In situ hybridizations with the NvemxLx probe on wild-type embryos often took $>2$ weeks to develop, and even then it was only detectable in very few cells and in only a few of the animals, arguing that it is expressed at low levels and that mRNA in situ hybridization is not sensitive enough to verify this gene. Relative expression strength can be 


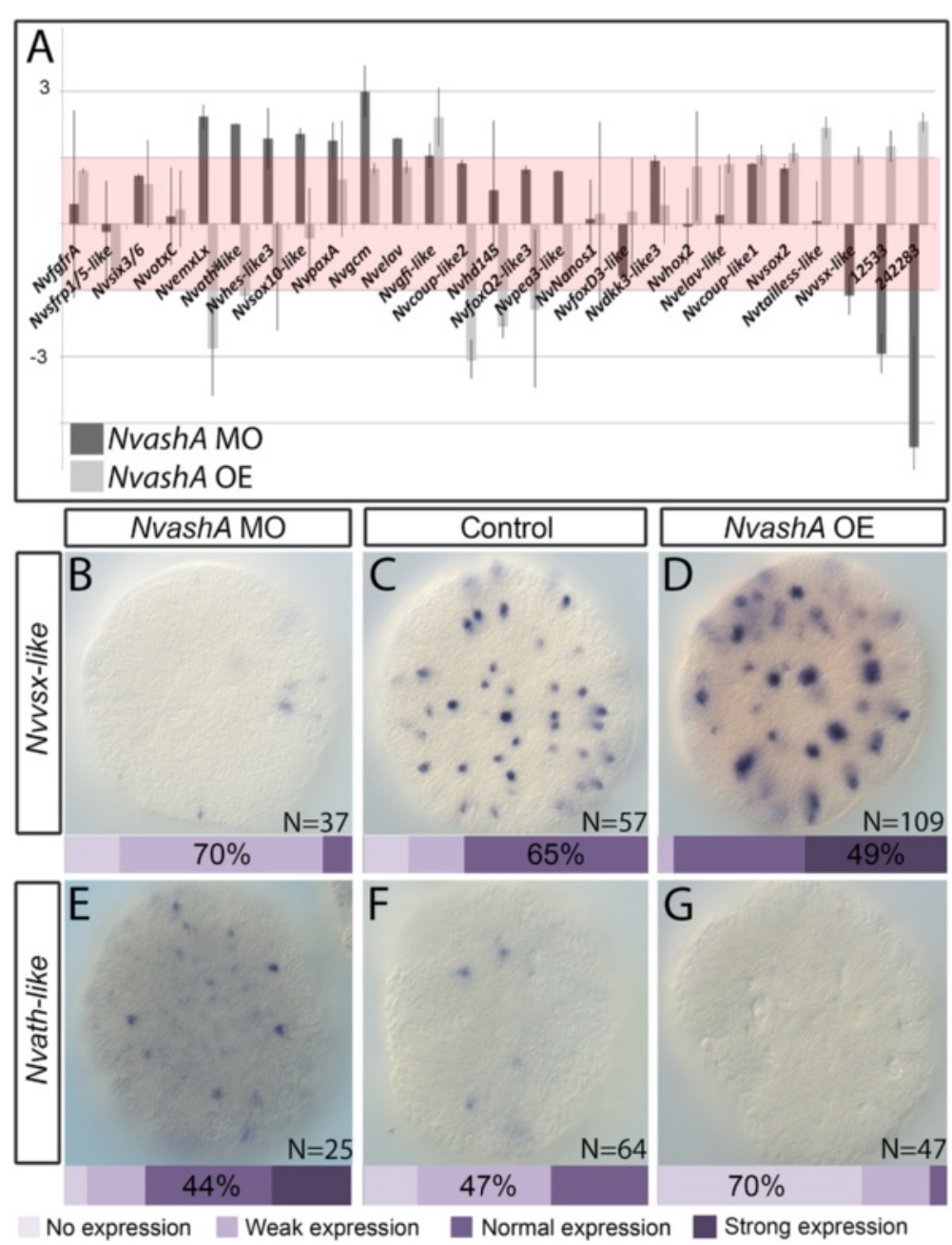

Fig. 8 NvashA regulates a subset of U0126-dependent salt-and-pepper expressed genes. a Relative fold change calculated from quantitative polymerase chain reaction analysis of triplicate injections of the NvashA morpholino $(M O)$ versus a control MO (dark gray bars), or NvashA:venus-injected versus control venus-injected animals (light gray bars). Broadly expressed NvfgfRa, Nvsfrp1/5, Nvsix3/6, and NvotxC regional patterning genes were included as controls. Two positive control genes, 12533 and 242283, are included. The remaining genes are the salt-and-pepper expressed genes identified by the U0126 microarray. The red box indicates a region between $1.5 \times$ and $-1.5 \times$ fold change that corresponds to an insignificant change in expression. The reciprocal phenotypes observed in NvashA MO and NvashA:venus mRNA-injected animals for Nvvsx-like (b-d) and Nvath-like $(\mathbf{e}-\mathbf{g})$ were confirmed by mRNA in situ hybridization. Embryos were classified and quantified as the percent having normal expression, weak expression, or no expression. The phenotypic class with the highest percentage of embryos is indicated. All embryo images are at the early gastrula stage. All images are ectodermal focal planes of aboral views. Error bars represent standard error

inferred by the qT value obtained for any given gene during $\mathrm{qPCR}$ analysis. Essentially, qT values above 35 are often associated with genes that are not expressed. We consistently obtained qT values of 32-33 for NvemxLx, arguing that it is in fact very weakly expressed. Although we could not confirm NvemxLx by mRNA in situ hybridization, it is likely a negative target of NvashA because it reproducibly showed reciprocal changes in expression in response to an increase or decrease in NvashA function (Fig. 8a). The remaining putative negative targets did not show reduced expression levels in NvashA:venus mRNA-injected animals, and with exception to Nvsox10-like, their increased expression in NvashA morphant animals could not be confirmed by mRNA in situ hybridization (Additional file 13: Figure S8; data not shown). The increased expression of Nvelav1 in NvashA morphants was somewhat surprising, because it has already been shown to be a positive target of NvashA when assayed at later stages [11, 36]. However, NvashA only regulates a subset of Nvelav1positive neurons [9-11], and thus we suspect that NvashA does not play a significant, if any, role in Nvelav1 regulation at this early time point. We conclude that Nvath-like, NvemxLX, and Nvsox10-like are normally 
suppressed by NvashA at embryonic stages, and that NvashA is sufficient to suppress expression of Nvath-like and NvemxLX but not Nvsox10-like.

\section{Discussion \\ Preliminary gene regulatory network and model describing NvashA-dependent neurogenesis in the embryonic ectoderm}

Based on previous observations and data presented here, we propose a model for and preliminary gene regulatory network describing NvashA-dependent neurogenesis in the early embryonic ectoderm of Nematostella (Fig. 9a, b). Based on U0126 phenotypes, we hypothesize that one or more not yet identified kinase signaling cascade(s) acting through MEK provides a global cue that is necessary for neural fates. It is not yet clear if all cells are competent to respond to this cue. However, it appears that the number of competent cells is greater than the number of cells that become neuralized, because evidence suggests that Notch signaling integrates the global cue to restrict a subset of cells to become Nvath-likeand NvsoxB(2)-positive neural progenitor cells (Fig. 9). Interestingly, we saw no evidence that either Nvnotch or Nvdelta were affected by U0126 treatment (Additional file 5: Table S2), which suggests that the refining activity of Nvnotch is independently controlled. It is not clear which, if either, transcription factor [Nvath-like or $N v \operatorname{sox} B(2)]$ is nearer the top of the neural cascade. $N v \operatorname{sox} B(2)$ is expressed maternally (Additional file 2: Figure $\mathrm{S} 1$ ), and both genes appear to be upregulated at approximately the same time in normal development (Additional file 2: Figure S1; Additional file 10: Figure S5). However, increased Nvath-like expression accumulates before $N v \operatorname{soxB}(2)$ increases in animals with inhibited Notch activity [9]. Additionally, $N v \operatorname{soxB}(2)$ and NvashA co-expression can be observed in post-mitotic cells, whereas Nvath-like and NvashA double-positive cells are never observed [9]. Post-mitotic neurons do not appear to express Nvath-like and they lose NvsoxB2 expression. Lineage-specific pro-differentiation neural markers such as NvashA are not expressed until post-mitotic stages. The observation that NvashA suppresses Nvathlike expression suggests that one of its functions is to inhibit neural progenitor identity. This is contrary to reported interactions for Nvath-like and NvashA [9]. However, the previous study assessed phenotypes at later time points, and thus cannot account for potential phenotypes arising due to sustained loss of a key neurogenic gene causing system-wide defects. Here we look closer at the onset of neurogenesis, which provides less time for potential nonspecific phenotypes to arise. Certainly further efforts are needed to clarify this point. Nva$\operatorname{sh} A$ also promotes the expression of distinct individual neuronal subtype markers. The mechanism by which subtype markers are regulated is still unclear, but it is likely that regionally expressed oral-aboral patterning genes and the temporal window in which neural progenitors/neurons are born likely contribute to neural patterning $[11,19,21]$.

It is not clear how only a subset of the $N v \operatorname{soxB}(2)$ and Nvath-like double-positive cells give rise to NvashA-expressing cells. NvsoxB(2), Nvath-like, and NvashA expression do not appear to be restricted to a distinct spatial domain $[9,11,41]$. One hypothesis is that progenitors give rise to different daughter cells with distinct identities, and that NvashA defines one such identity. This idea would be consistent with temporal patterning observed for neural progenitor lineages in Drosophila [58]. Alternatively, the time and position at which a progenitor is born might determine its identity and subsequently the identity of the neurons it generates [59, 60]. Regardless, functional studies support a much broader role for the progenitor marker $N v \operatorname{soxB}(2)$, and suggest that some additional mechanism is acting to restrict $N v a s h A$ expression to a subset of the $N v \operatorname{sox} B(2)$ positive progenitors.

\section{Additional putative neural genes identified here}

We have identified 18 new genes expressed early (in late blastula/early gastrula) stages that are candidate neural regulators or neural subtype markers. The earliest expressed gene in our temporal analysis was Nvath-like, which had been previously identified as an early-acting neurogenic gene. Two genes turned on slightly after Nvath-like were Nvhes3-like and NvfoxD3-like. Previous studies suggest that Nvhes3-like is not sufficient to regulate neurogenesis at this stage, but it cannot yet be ruled out as a neural regulator because efficient Nvhes3-like knockdowns are not yet reported [36]. The next two genes to be expressed were Nvsox2 and Nvfoxq2-like. Again, the broad expression of Nvsox 2 was similar to that of NvfoxD3-like and suggests that it may act broadly to regulate neurogenesis. Nvfoxq2-like genes displayed limited expression in the aboral region, suggesting their neurogenic potential is limited to neurons arising from that domain. The late-onset genes displayed both broad and restricted expression patterns, suggesting that at least some of these genes have roles regulating distinct neural subtypes. This is supported by the inclusion of NvashA as a late-onset gene. However, it must be noted that neurogenesis in Nematostella is a continuous process and late onset alone is not sufficient to suggest that a gene acts at the later steps in neurogenesis. Regardless, we have identified a number of putative neural genes, and future work will allow us to both determine which genes are definitively neural, and where each gene fits into the regulatory networks describing the earliest born neural subtypes in Nematostella. 


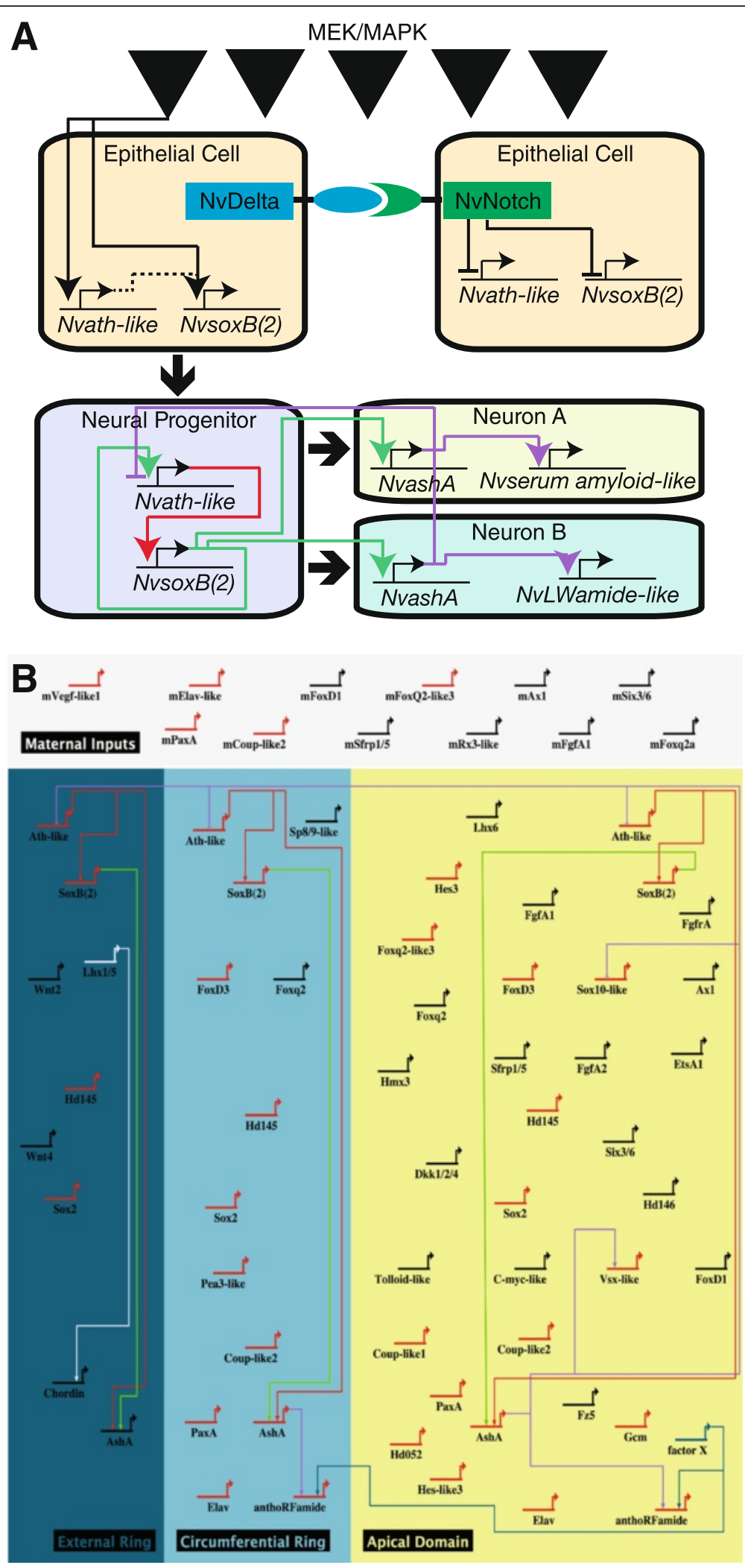

Fig. 9 Model of NvashA-dependent embryonic neurogenesis. a Model describing NvashA-dependent neurogenesis at early gastrula stage. Boxes represent indicted cell types. Solid regulatory lines represent published observations, and dashed lines represent likely regulatory interactions. b Biotapestry diagram of GRN 


\section{Neural induction in Nematostella}

MAPK/MEK activity is required for the expression of key neural progenitor markers, suggesting that it might be a component of neural induction in Nematostella. Loss of neurogenesis following treatment with U0126 implies that a positive neurogenic cue is disrupted by U0126 treatment. It is tempting to speculate that FGF signaling may be responsible for neural induction, because FGF is a positive regulator of neural induction during vertebrate development [15] and MEK activity has been described to be downstream of FGF signaling in other systems. However, we report no phenotype when the broadly expressed NvfgfRa receptor was knocked down (Fig. 7). However, we still suspect that some MAPK signaling through MEK is necessary for neurogenesis. $\alpha-$ phospho-Erk staining was detected throughout the embryonic ectoderm, and this staining was reduced in U0126treated animals (Fig. 2). Recently, MAPK signaling was shown to positively promote neurogenesis in salamander animal cap explants, in part by inhibiting BMP responsiveness due to decreased smad1 expression [13]. However, expression of the putative neural marker Nvgata is not sensitive to the level of Nvbmp2/4 in Nematostella gastrula [23]. Thus, the potential mechanism by which MAPK/MEK signaling promotes neural fates is not yet understood in Nematostella.

\section{Possibly multiple neural inductive events during Nematostella neurogenesis}

Multiple observations raise the hypothesis that distinct mechanisms may be necessary for neurogenesis in different spatiotemporal windows in Nematostella. First, Nvbmp2/4 does not appear to impact neurogenesis at early embryonic stages, but expression of neural markers such as Nvelav1 and NvashA are sensitive to the levels of $N v b m p 2 / 4$ and $N v b m p 5 / 8$ in planulae $[22,23]$. Second, NvashA was not detected in U0126-treated embryos at early gastrula stages, but animals that were allowed to continue developing in the presence of U0126 begin to show NvashA expression, albeit in very few cells (Additional file 12: Figure S7). Third, very few NvLWamide-positive and NvanthoRFamide-positive cells were able to form in U0126-treated animals, which suggests that a subset of $N v L W a m i d e$ and NvanthoRFamide neurons may be U0126-independent. The potential for multiple neural programs acting in Nematostella suggest that efforts to isolate specific neurons born in unique spatiotemporal windows as well developing conditional alleles to investigate later time points in isolation will improve our understanding of neurogenesis in Nematostella.

\section{Patterning genes identified in the U0126 microarray}

In addition to the genes with a neural-like expression pattern, our forward molecular approach identified $\sim 40$ genes downstream of MEK that were expressed at the animal pole, including genes expressed in the presumptive endomesoderm and genes expressed at the aboral pole. These data suggest that MAPK acts broadly in the embryo to regulate germ layer specification as well as regional identities associated with axial patterning. UO126 treatment has been reported to suppress formation of the apical tuft at the aboral pole of the larva, likely via inhibition of NvFGFRa-mediated MAPK signaling [24, 26]. However, early embryonic patterning of the aboral domain has been largely understudied, and preliminary data suggest that distinct mechanisms act at early (embryonic) and late (larval) stages of aboral patterning [19]. Additional FGF receptors and ligands are detected in the oral domain of Nematostella, suggesting that FGFmediated MAPK signaling normally regulates U0126 targets identified that display oral/animal pole expression [25]. However, targeted gene-specific knockdowns will be important to further determine exact mechanisms by which MEK activity impacts aboral and/or germ layer specification.

\section{Conclusions}

Our data indicate MAPK signaling is necessary for neurogenesis in the embryonic ectoderm of Nematostella. Our work also built upon previous observations to improve our understanding of the molecular mechanisms underlying cnidarian neurogenesis and described two transgenes that describe distinct neural subtypes in Nematostella. Lastly, we identified $\sim 120$ signaling molecules and transcription factors that act downstream of MEK in MAPK signaling. Future characterization of the genes will provide critical cues about the early patterning mechanisms acting during Nematostella development, which will be important to allow improved understanding about the origin and evolution of neurogenesis, axial patterning, and endomesoderm specification.

\section{Methods}

\section{Culture and spawning of Nematostella vectensis}

Adult Nematostella were cultivated either at the Kewalo Marine Laboratory/PBRC of the University of Hawaii (USA), the Whitney Laboratory for Marine Bioscience of the University of Florida (USA), the Institute for Research on Cancer and Aging of the University of Nice-Sophia-Antipolis (FRA), or Lehigh University (USA) according to the protocol described in [45]. Males and females were kept in separate glass bowls $(250 \mathrm{ml})$ in $1 / 3 \times$ seawater (salinity: $12 \mathrm{pp}$ ) [5] at $17{ }^{\circ} \mathrm{C}$ in the dark and water was changed weekly. Animals were fed three/ four times a week with pieces of oysters or brine shrimps. Manipulating the light cycle induced spawning and oocytes and sperm were collected separately [4]. 
The gelatinous mass around the eggs was removed with $4 \%$ L-Cystein in $1 / 3 \times$ seawater before fertilization and then washed three times with $1 / 3 \times$ seawater. For the simultaneous development of the embryos, all oocytes were fertilized in glass dishes at the same time with $0.5 \mathrm{ml}$ of diluted sperm. Fertilized eggs were kept in dark in filtered $1 / 3 \times$ seawater at $17{ }^{\circ} \mathrm{C}$ until the desired stage.

\section{U0126 and SU5402 treatments}

The MEK (U0126, Sigma, \# U120) and FGF (SU5402, Sigma, \# SML0443) antagonists were dissolved at a stock concentration of $10 \mathrm{mM}$ in DMSO and added at to $1 / 3 \times$ filtered seawater to generate final concentrations as indicated. For SU5402, analysis was done at a final concentration of $20 \mu \mathrm{M}$, and for U0126 most experiments were conducted at a final concentration of $15 \mu \mathrm{M}$. Unless indicated, embryos were treated with the drug directly after fertilization and kept in the dark at $17{ }^{\circ} \mathrm{C}$. If needed, U0126 was replaced every $24 \mathrm{~h}$ with fresh solutions to maintain activity. Treatments were compared to DMSO-treated control embryos. Embryos were fixed for in situ hybridization and morphological analysis at indicated stages. mRNA of embryos was extracted 24 hpf (late blastula stage) from two distinct biological replicates for microarray analysis.

\section{RNA extraction, quantitative PCR, and microarray analysis}

RNA extraction, qPCR, and microarray analysis were performed following protocols described in [46]. RNA for qPCR and microarray analysis was isolated with TriPure (Roche, \#11667157001) or TRIzol (Invitrogen, \#15596-026) according to the manufacturer's instructions, and genomic contamination removed using RNase-free DNase (Qiagen, \#79254) for $15 \mathrm{~min}$ at $37^{\circ} \mathrm{C}$. The total amount of RNA was quantified with a NanoDrop 2000 spectrophotometer (Thermo Scientific) and the quality analyzed with a Bioanalyzer 2100 (Agilent Technologies Inc.). To generate cDNA, $1 \mu \mathrm{g}$ of total RNA was used with the Advantage RT-PCR kit (Clontech, \#639506) for qPCR analysis. For the fine-scale temporal analysis, total RNA was extracted from the following stages (in hpf): 0, 2, 4, 6, 8, 10, 12, 14, 16, 18, $20,24,28,32,40$, and 48 . qPCR analysis using a LightCycler 480 (Roche) utilizing LightCycler 480 SYBR Green 1 Master mix (Roche, \#04887352001) was carried out as described previously [11]. Efficiencies for each gene-specific primer pair was determined using a fivefold serial dilution series and only primers with an efficiency ranging from 1.8 to 2.15 were used for further analysis (Additional file 14: Table S6). The housekeeping genes Nvactin and/or Nvgadph were used to normalize relative fold changes between control and manipulated embryos. Each qPCR analysis was repeated on at least three independent biological replicates and changes were analyzed using a Student's $t$ test. Microarray analysis was conducted by sending $20 \mu \mathrm{g}$ of total RNA (RIN value $>8$ ) to NimbleGen (Iceland) for further cDNA synthesis, labeling, and array hybridization. Two replicates were sent for each control and U0126-treated animals were sent. The 4-plex microarray (72,000 features) is an oligonucleotide-based chip version, custom designed and produced by NimbleGen Systems (Roche). Array data are available from http://www.ebi.ac.uk/arrayexpress/experiments/E-MTAB-4831/. Gene expression levels were normalized in the Nimblescan software according to $[47,48]$ and fold changes calculated by comparing expression values from control and treated embryos. Array results were screened based on the provided genome annotations assigned to each array spotID. If no clear blast hit or gene information was assigned to the prediction gene model from the Joint Genome Institute, we retrieved the genomic sequences from http://genome.jgi-psf.org/Nemve1/Nemve1.home.html for the given gene and performed manual BLAST (BLASTX) searches [49] against the NCBI database to determine the nature of the predicted gene product. All sequences from genes of interest have been used for BLAST analysis to confirm their nature and to determine previously published genes.

\section{In situ hybridization, pERK, actin, and nuclear staining}

Previously described gene sequences were used to subclone into pGemT (Promega, \#A3600) from mixed stage cDNA. All other sequences used in this study were isolated in the course of a microarray analysis. Genome predictions as well as expressed sequence tag sequence information were combined to design primers (Additional file 15: Table S7) that allowed the amplification and cloning of genes between $0.5 \mathrm{~kb}$ and $2 \mathrm{~kb}$ as described above. Accession numbers for all analyzed genes in this study can be found in Additional file 6: Table S3 and Additional file 7: Table S4. Embryo fixation, probe synthesis, and in situ hybridization were performed as previously described [42]. The MegaScript Transcription Kit (Ambion) was used to synthesize $0.5-2 \mathrm{~kb}$ digoxigenin (DIG)-labeled (Roche, \#11573152910) riboprobes. Hybridization of riboprobes $(1 \mathrm{ng} / \mu \mathrm{l})$ was carried out at $62{ }^{\circ} \mathrm{C}$ in $50 \%$ formamide hybe buffer and visualization of the labeled probe was performed using NBT/BCIP as a substrate for the alkaline phosphatase-conjugated anti-DIG antibody (Roche, \#11093274910). To analyze embryonic and larval morphology, we used Biodipy FL Phallacidin (Molecular Probes/ Invitrogen, \#B607) and propidium iodide (Sigma, \#81845) to stain $\mathrm{f}$-actin and the cell nuclei respectively as described previously [61]. To analyze embryonic localization of activated ERK, we used a monoclonal antibody that recognizes a phosphorylated epitope of the activated form of ERK [Phospho-p44/42 MAPK (Erk1/2) (Thr202/Tyr204); 
Cell Signaling, \# 4370)]. Antibodies were diluted at 1:200 in blocking solution (PBT (Phosphate Buffered Saline + $0.1 \%$ Tween) $+10 \%$ normal goat serum) overnight at $4{ }^{\circ}$ C. Following six washes in PBT, embryos were incubated with the secondary antibody (anti-rabbit Ig), and diluted at $1: 250$ for at least $4 \mathrm{~h}$ to overnight at $4{ }^{\circ} \mathrm{C}$ on a shaking rocker. Phosphate-buffered saline (PBS) was used for washes between antibodies. Specimens were mounted in $80 \%$ glycerol.

\section{Imaging}

In situ hybridization images were taken on either a Zeiss AxioScop 2 mounted with an Axiocam camera triggered by Axiovision software (Carl Zeiss), a Zeiss Axio Imager A2 mounted with a Canon 6D triggered by Canon professional software, or a Nikon NTi using a Nikon DSRi2 color camera and the Elements software (Nikon). All expression patterns described here have been submitted to Kahi Kai, a comparative invertebrate gene expression database [62] hosted at http://www.kahikai.org/ index.php?content=genes. Scoring of treatment phenotypes was performed on either a Zeiss Z-1 Axio imager or a Zeiss Axio Imager A2 microscope and confocal imaging was conducted on either a Zeiss LSM710 or Zeiss LSM Exciter microscope running the LSM ZEN software (Carl Zeiss). Fluorescent images were false-colored. The fluorescent channels were merged using ImageJ (http:// rsbweb.nih.gov/ij/) and cropped to final size in Photoshop Cs6 (Adobe Inc.). Confocal images for Fig. 5 were processed using Imaris 8.1 (Bitplane).

\section{Microinjection of mRNA and morpholinos}

NvashA:venus or venus mRNA was injected into embryos at $150 \mathrm{ng} / \mu \mathrm{l}$ as previously described [6, 11, 36]. Antisense translation blocking MO against Nvfgfra [24] and NvashA [11] (GeneTools Inc.; Philomath, OR, USA) and a control MO (5' AATAAAAAGAATGCCCCCTCACCTCT $3{ }^{\prime}$ ) with no known targets in the predicted Nematostella genome were injected at $1 \mathrm{mM}$ concentrations.

\section{Transgenic strain generation}

To generate transgenic animals we amplified genomic DNA from position Scaffold21: 1349994-1347681 for Nv239910 (serum amyloid A-like) and from Scaffold60: 1049346-1046951 for Nv242283 (NvLWamide-like). Numbers correspond to genomic positions available in the Nematostella genome version $1.0 \mathrm{http}: / /$ genome.jgi.doe.gov/Nemve1/Nemve1.home.html. We cloned each fragment into the pNvT vector in front of the mcherry coding sequence as previously reported [37]. Animals were injected as previously reported [37], and stable F1 lines were identified by screening for fluorescence and outcrossing to wild-type animals.

\section{Additional files}

Additional file 1: Table S1. Dose-dependent effects of U0126 on Nvsprouty, Nvbrachyury, and Nv-fgfAl expression. Dose-dependent effects of U0126 analyzed by in situ hybridization. Analyzed U0126 concentration as indicated in Row 1 and number of embryos with phenotype scored based on expansion/reduction of the domain of expression as indicated in the column on the right. (XLS $33 \mathrm{~kb}$ )

Additional file 2: Figure S1. Summary of NvsoxB(2) RNA-seq data. Plot used data obtained from [33] from two duplicate RNA-seq data sets that generated transcriptomes over the first $19 \mathrm{hpf}$. Black trace shows average of two replicates. (JPG $471 \mathrm{~kb}$ )

Additional file 3: Figure S2. Changes in NvashA, Nvath-like, and NvsoxB2 expression following drug treatments or in with increased Notch activity. mRNA in situ hybridization for $\operatorname{NvsoxB(2)}(\mathbf{A})$ and NvashA (B) in DMSO-treated control animals grown to early planula stages (48 hpf at $\left.22^{\circ} \mathrm{C}\right)$. NvsoxB(2) $\left(\mathbf{A}^{\prime}\right)$ and NvashA ( $\left.\mathbf{B}^{\prime}\right)$ expression in same stage animal treated with U0126 from 24 to 48 hpf or treated with SU5402 from 24 to 48 hpf $\left(\mathbf{B}^{\prime \prime}\right)$. mRNA in situ expression of Nvath-like (C) and NvsoxB(2) (D) in control embryos injected with the venus mRNA. Expression of Nvath-like $\left(\mathbf{C}^{\prime}\right)$ and $\operatorname{NvsoxB(2)}\left(\mathbf{D}^{\prime}\right)$ in animals injected with Nvnotch/CD:venus (the intracellular domain of the Notch receptor), which has been previously shown to hyperactivate Notch signaling. Embryos were classified and quantified as the percent having normal expression, weak expression, or no expression. The phenotypic class with the highest percentage of embryos is indicated. In C and D, the main figure panels are ectodermal focal planes, and insets show deeper focal planes used to confirm embryonic stage. All images are of lateral views with the oral side to the left. (TIF 34682 kb)

Additional file 4: Figure S3. NvAshA:Venus localization in U0126treated animals. NvAshA:Venus protein was detected at high levels and with strong nuclear localization in U0126-treated animals. (TIF 16187 kb)

Additional file 5: Table S2. Array data for NvsoxB(2), Nvnotch, Nvdelta, and neural genes used for transgenes or controls in $\mathrm{PPCR}$ experiments. (XLSX $38 \mathrm{~kb}$ )

Additional file 6: Table S3. Genes with at least a 2-fold change after U0126 treatments based on our array analysis. (XLSX 3983 kb)

Additional file 7: Table S4. Selection of 22 genes upregulated after U0126 treatment. Selected genes that were significantly $(P<0.05)$ at least 2 -fold upregulated by $\cup 0126$ treatments. SpotID was the genome protein model ID (JGI) used for the array design. The gene name is based on the best BLAST hit and if available the previously published name(s) is used. Abbreviations are indicated in the table legend at the bottom. References are included in the main text. (XLSX $27 \mathrm{~kb}$ )

Additional file 8: Table S5. Selection of 100 genes downregulated after $\cup 0126$ treatments. Selected genes that were significantly $(P<0.05)$ at least 2-fold downregulated by U0126 treatments. SpotID was the genome protein model ID (JGI) used for the array design. The gene name is based on the best BLAST hit and if available the previously published name(s) is used. Color code and abbreviations are indicated in the table legend at the bottom. References are included in the main text. (XLSX $37 \mathrm{~kb}$ )

Additional file 9: Figure S4. Summary of animal hemisphere and aboral expression genes identified by $U 0126$ array. mRNA in situ patterns are included in a manuscript currently in preparation. (PDF 39 kb)

Additional file 10: Figure S5. Gene expression analyzed by quantitative polymerase chain reaction. Salt-and-pepper expressed genes as represented in Fig. 6. High-density gene expression profiles are represented by charts for all genes expressed at the blastula stage [24 hours post fertilization (hpf)] and/or gastrula stage (48 hpf) analyzed in this study. The $y$-axis indicates the relative fold change compared to unfertilized eggs. The $x$-axis indicates developmental time in hpf. Gene names as indicated in the top left corner and the Cp value in unfertilized eggs is indicated in the top right corner of each panel and was used to determine the presence of maternal transcripts in Fig. 6 (Cp > 34.00). Cp corresponds to the crossing point, also known as the cycle threshold $(C t)$ value. (PDF 599 kb)

Additional file 11: Figure S6. Summary temporal gene expression analysis. Summarized results of the temporal high density profiling (qPCR) 
used to determine the presence of maternal transcripts and significant zygotic upregulation of a given gene expressed in individual cells of the ectoderm. n.d. Not determined. *Genes that have been identified and their spatial blastula and gastrula expression patterns characterized elsewhere (see Additional file 6: Table S3, Additional file 7: Table S4 and Additional file 8: Table S5). (PDF $62 \mathrm{~kb}$ )

Additional file 12: Figure S7. NvashA expression in animals with varied regiments of U0126 treatment. (A) NvashA expression in control animals, or in animals treated with U0126 continuously for 48 hours, or from 24 to $48 \mathrm{hpf}$. Unlike early stages when no NvashA expression could be detected (Fig. 3), NvashA expression was ultimately detected in U0126treated animals by $48 \mathrm{hpf}$. Treatment with $\cup 0126$ from 24 to $48 \mathrm{hpf}$ reduced NvashA expression, but NvashA could be detected in many cells, albeit at reduced levels. (B) Levels of NvashA and Nvfgfal as detected by qPCR at late gastrula stage $\left(48 \mathrm{hpf}\right.$ at $\left.17^{\circ} \mathrm{C}\right)$ in animals injected with the Nvfgfra MO or treated with U0126 or SU5402 from 24 to 48 hpf. Relative expression levels are compared to control MO- or DMSO-treated animals respectively. The red box defines 1.5 to -1.5 fold change region. Error bars are standard error. (TIF $11166 \mathrm{~kb}$ )

Additional file 13: Figure S8. NvashA regulation of target genes in the embryonic ectoderm. Gene expression in NvashA morphants (A-C), control morpholino (D-F), and NvashA mRNA injected $(\mathbf{G}, \mathbf{H})$. Quantification below each image represents percent of embryos in each phenotypic class (see key in figure). All images except $\mathrm{C}$ and $\mathrm{F}$ are aboral views. $\mathrm{C}$ and $\mathrm{F}$ are oral views. Embryos were classified and quantified as the percent having normal expression, weak expression, or no expression.. The phenotypic class with the highest percentage of embryos is indicated. (TIF $21122 \mathrm{~kb}$ )

Additional file 14: Table S6. Primer pairs used in this study for $\mathrm{qPCR}$ analysis. (XLS $53 \mathrm{~kb}$ )

Additional file 15: Table S7. Primer pairs used in this study for gene cloning. (XLS $73 \mathrm{~kb}$ )

\section{Acknowledgments}

We would like to acknowledge Dr Uli Technau for providing reagents necessary to generate transgenic lines as well as the reviewers for providing helpful feedback.

\section{Authors' contributions}

$M J L, E R$, and MQM conceived of the study, performed experiments, analyzed data, and drafted the manuscript. ARA, BS, HJ, TC and JH generated, collected, and analyzed data. All authors read and approved the final manuscript.

\section{Competing interests}

The authors declare that they have no competing interests.

\section{Author details}

'Department of Biological Sciences, Lehigh University, Bethlehem, PA, USA. ${ }^{2}$ The Whitney Marine Laboratory for Marine Science, University of Florida, St. Augustine, Florida, USA. ${ }^{3}$ Université Nice Sophia Antipolis UMR 7284, CNRS UMR 7284, INSERM U1081, Institute for Research on Cancer and Aging, Nice, France.

Received: 20 March 2016 Accepted: 4 July 2016

Published online: 01 August 2016

\section{References}

1. Layden MJ, Rentzsch F, Röttinger $\mathrm{E}$. The rise of the starlet sea anemone Nematostella vectensis as a model system to investigate development and regeneration. WIREs Dev Biol. 2016;1-21.

2. Putnam NH, Srivastava M, Hellsten U, Dirks B, Chapman J, Salamov A, et al. Sea anemone genome reveals ancestral Eumetazoan gene repertoire and genomic organization. Science. 2007;317:86-94.

3. Stefanik DJ, Friedman LE, Finnerty JR. Collecting, rearing, spawning and inducing regeneration of the starlet sea anemone, Nematostella vectensis. Nat Protoc. 2013;8:916-23.

4. Fritzenwanker $\mathrm{JH}$, Technau U. Induction of gametogenesis in the basal cnidarian Nematostella vectensis (Anthozoa). Dev Genes Evol. 2002;212:99-103.
5. Hand C, Uhlinger KR. The Culture, sexual and asexual reproduction, and growth of the sea anemone Nematostella vectensis. Biol Bull. 1992;182:169-76.

6. Layden MJ, Röttinger E, Wolenski FS, Gilmore TD, Martindale MQ. Microinjection of mRNA or morpholinos for reverse genetic analysis in the starlet sea anemone, Nematostella vectensis. Nat Protoc. 2013;8: 924-34.

7. Marlow HQ, Srivastava M, Matus DQ, Rokhsar D, Martindale MQ. Anatomy and development of the nervous system of Nematostella vectensis, an anthozoan cnidarian. Devel Neurobio. 2009;69:235-54.

8. Nakanishi N, Renfer E, Technau U, Rentzsch F. Nervous systems of the sea anemone Nematostella vectensis are generated by ectoderm and endoderm and shaped by distinct mechanisms. Development. 2012:139:347-57.

9. Richards GS, Rentzsch F. Regulation of Nematostella neural progenitors by SoxB, Notch and bHLH genes. Development. 2015;142:3332-42.

10. Richards GS, Rentzsch F. Transgenic analysis of a SoxB gene reveals neural progenitor cells in the cnidarian Nematostella vectensis. Development. 2014;141:4681-9.

11. Layden MJ, Boekhout M, Martindale MQ. Nematostella vectensis achaetescute homolog NvashA regulates embryonic ectodermal neurogenesis and represents an ancient component of the metazoan neural specification pathway. Development. 2012;139:1013-22.

12. Wilson SI, Edlund T. Neural induction: toward a unifying mechanism. Nat Neurosci. 2001;4:1161-8.

13. Hurtado C, De Robertis EM. Neural induction in the absence of organizer in salamanders is mediated by MAPK. Dev Biol. 2007;307:282-9.

14. Gaulden J, Reiter JF. Neur-ons and neur-offs: regulators of neural induction in vertebrate embryos and embryonic stem cells. Hum Mol Genet. 2008;17:R60-6

15. Stern CD. Neural induction: old problem, new findings, yet more questions. Development. 2005;132:2007-21

16. Bertrand V, Hudson C, Caillol D, Povovici C, Lemair P. Neural tissue in Ascidian embryos is induced by FGF9/16/20, acting via a combination of maternal GATA and Ets transcription factors. Cell. 2003:115:615-27.

17. Nordström $U$, Jessell TM, Edlund T. Progressive induction of caudal neural character by graded Wnt signaling. Nat Neurosci. 2002:5:525-32.

18. Marchal L, Luxardi G, Thome V, Kodjabachian L. BMP inhibition initiates neural induction via FGF signaling and Zic genes. PNAS. 2009;106:17437-42.

19. Sinigaglia $C$, Busengdal $H$, Leclère $L$, Technau $U$, Rentzsch $F$. The bilaterian head patterning gene six3/6 controls aboral domain development in a cnidarian. PLoS Biol. 2013;11:e1001488.

20. Robinson R. Ciliate genome sequence reveals unique features of a model eukaryote. PLoS Biol. 2006;4:e304.

21. Marlow H, Matus DQ, Martindale MQ. Ectopic activation of the canonical wnt signaling pathway affects ectodermal patterning along the primary axis during larval development in the anthozoan Nematostella vectensis. Dev Biol. 2013:380:324-34.

22. Watanabe H, Kuhn A, Fushiki M, Agata K, Ozbek S, Fujisawa T, et al. Sequential actions of $\beta$-catenin and Bmp pattern the oral nerve net in Nematostella vectensis. Nat Commun. 2014;5:5536-14.

23. Saina M, Genikhovich G, Renfer E, Technau U. BMPs and chordin regulate patterning of the directive axis in a sea anemone. Proc Natl Acad Sci USA. 2009:106:18592-7.

24. Rentzsch F, Fritzenwanker JH, Scholz CB, Technau U. FGF signalling controls formation of the apical sensory organ in the cnidarian Nematostella vectensis. Development. 2008;135:1761-9.

25. Matus DQ, Thomsen GH, Martindale MQ. FGF signaling in gastrulation and neural development in Nematostella vectensis, an anthozoan cnidarian. Dev Genes Evol. 2007;217:137-48.

26. Sinigaglia C, Busengdal $H$, Lerner A, Oliveri P, Rentzsch F. Molecular characterization of the apical organ of the anthozoan Nematostella vectensis. Dev Biol. 2015:398:120-33.

27. Favata MF, Horiuchi KY, Manost EJ, Daulario AJ, Stradley DA, Fesser WS, et al. Identification of a novel inhibitor of mitogen-activated protein kinase kinase*. J Biol Chem. 1998;273:18623-32.

28. Röttinger $\mathrm{E}$, Dahlin P, Martindale MQ. A framework for the establishment of a cnidarian gene regulatory network for "endomesoderm" specification: the inputs of ß-catenin/TCF signaling. PLoS Genet. 2012;8:e1003164.

29. Scholz CB, Technau U. The ancestral role of Brachyury: expression of NemBra1 in the basal cnidarian Nematostella vectensis (Anthozoa). Dev Genes Evol. 2003;212:563-70. 
30. Hacohen N, Kramer S, Sutherland D, Hiromi Y, Krasnow MA. sprouty encodes a novel antagonist of FGF signaling that patterns apical branching of the Drosophila airways. Cell. 1998;92:253-63.

31. Yung $Y$, Dolginov $Y$, Zhong $Y$, Rubinfeld $H$, Michael $D$, Hanoch $T$, et al. Detection of ERK activation by a novel monoclonal antibody. FEBS Lett. 1997:408:292-6.

32. Fischer AHL, Mozzherin D, Eren AM, Lans KD, Wilson N, Cosentino C, et al. SeaBase: a multispecies transcriptomic resource and platform for gene network inference. Integr Comp Biol. 2014;54:250-63.

33. Tulin S, Aguiar D, Istrail S, Smith J. A quantitative reference transcriptome for Nematostella vectensis early embryonic development: a pipeline for de novo assembly in emerging model systems. Evodevo. 2013;4:16-1.

34. Helm RR, Siebert S, Tulin S, Smith J, Dunn CW. Characterization of differential transcript abundance through time during Nematostella vectensis development. BMC Genomics. 2013:14:266.

35. Shimojo H, Ohtsuka T, Kageyama R. Oscillations in notch signaling regulate maintenance of neural progenitors. Neuron. 2008;58:52-64.

36. Layden MJ, Martindale MQ. Non-canonical Notch signaling represents an ancestral mechanism to regulate neural differentiation. Evodevo. 2014:5:1-14

37. Renfer E, Amon-Hassenzahl A, Steinmetz PRH, Technau U. A muscle-specific transgenic reporter line of the sea anemone, Nematostella vectensis. Proc Natl Acad Sci USA. 2010;107:104-8.

38. Srivastava M, Larroux C, Lu DR, Mohanty K, Chapman J, Degnan BM, et al. Early evolution of the LIM homeobox gene family. BMC Biol. 2010;8:4.

39. Ryan JF, Mazza ME, Pang K, Matus DQ, Baxevanis AD, Martindale MQ, et al. Pre-bilaterian origins of the hox cluster and the hox code: evidence from the sea anemone, Nematostella vectensis. PLoS One. 2007;2:e153-23.

40. Lee PN, Pang K, Matus DQ, Martindale MQ. A WNT of things to come: evolution of Wht signaling and polarity in cnidarians. Semin Cell Dev Biol. 2006;17:157-67.

41. Magie CR, Pang K, Martindale MQ. Genomic inventory and expression of Sox and Fox genes in the cnidarian Nematostella vectensis. Dev Genes Evol. 2005;215:618-30.

42. Martindale MQ. Investigating the origins of triploblasty: 'mesodermal' gene expression in a diploblastic animal, the sea anemone Nematostella vectensis (phylum, Cnidaria; class, Anthozoa). Development. 2004;131:2463-74.

43. Fritzenwanker $\mathrm{JH}$, Saina M, Technau U. Analysis of forkhead and snail expression reveals epithelial-mesenchymal transitions during embryonic and larval development of Nematostella vectensis. Dev Biol. 2004;275:389-402.

44. Matus DQ, Pang K, Daly M, Martindale MQ. Expression of Pax gene family members in the anthozoan cnidarian, Nematostella vectensis. Evol Dev. 2007:9:25-38.

45. Ryan JF, Burton PM, Mazza ME, Kwong GK, Mullikin JC, Finnerty JR. The cnidarian-bilaterian ancestor possessed at least 56 homeoboxes: evidence from the starlet sea anemone, Nematostella vectensis. Genome Biol. 2006;7:R64.

46. Kusserow A, Pang K, Sturm C, Hrouda M, Lentfer J, Schmidt HA, et al. Unexpected complexity of the Wnt gene family in a sea anemone. Nature. 2005;433:156-60.

47. Santagata S, Resh C, Hejnol A, Martindale MQ, Passamaneck YJ. Development of the larval anterior neurogenicdomains of Terebratalia transversa (Brachiopoda) provides insights into the diversification of larval apical organs and the spiralian nervous system. Evodevo. 2012;3:3.

48. Kumburegama S, Wijesena N, Xu R, Wikramanayake AH. Strabismusmediated primary archenteron invagination is uncoupled from Wnt/ $\beta$ catenin-dependent endoderm cell fate specification in Nematostella vectensis (Anthozoa, Cnidaria): implications for the evolution of gastrulation. Evodevo. 2011;2:2

49. Matus DQ, Magie CR, Pang K, Martindale MQ, Thomsen GH. The Hedgehog gene family of the cnidarian, Nematostella vectensis, and implications for understanding metazoan Hedgehog pathway evolution. Dev Biol. 2008; 313:501-18

50. Layden MJ, Meyer NP, Pang K, Seaver EC, Martindale MQ. Expression and phylogenetic analysis of the zic gene family in the evolution and development of metazoans. Evodevo. 2010;1:12

51. Marlow H, Roettinger E, Boekhout M, Martindale MQ. Functional roles of Notch signaling in the cnidarian Nematostella vectensis. Dev Biol. 2012; 362:295-308.

52. Yasuoka Y, Kobayashi M, Kurokawa D, Akasaka K, Saiga H, Taira M. Evolutionary origins of blastoporal expression and organizer activity of the vertebrate gastrula organizer gene $\mathrm{lhx}$ I and its ancient metazoan paralog Ihx3. Development. 2009;136:2005-14.
53. Extavour CG, Pang K, Matus DQ, Martindale MQ. vasa and nanos expression patterns in a sea anemone and the evolution of bilaterian germ cell specification mechanisms. Evol Dev. 2005;7:201-15.

54. Mazza ME, Pang K, Martindale MQ, Finnerty JR. Genomic organization, gene structure, and developmental expression of three Clustered otx genes in the sea anemone Nematostella vectensis. J Exp Zool. 2007;308B:494-506.

55. Reitzel AM, Tarrant AM. Nuclear receptor complement of the cnidarian Nematostella vectensis: phylogenetic relationships and developmental expression patterns. BMC Evol Biol. 2009;9:230.

56. Sullivan JC, Sher D, Eisenstein M, Shigesada K, Reitzel AM, Marlow H, et al. The evolutionary origin of the Runx/CBFbeta transcription factors-studies of the most basal metazoans. BMC Evol Biol. 2008;8:228.

57. Chourrout D, Delsuc F, Chourrout P, Edvardsen RB, Rentzsch F, Renfer E, et al. Minimal ProtoHox cluster inferred from bilaterian and cnidarian Hox complements. Nature. 2006;442:684-7.

58. Isshiki T, Pearson B, Holbrook S, Doe CQ. Drosophila neuroblasts sequentially express transcription factors which specify the temporal identity of their neuronal progeny. Cell. 2001;106:511-21.

59. Jacob J, Maurange C, Gould AP. Temporal control of neuronal diversity: common regulatory principles in insects and vertebrates? Development. 2008;135:3481-9.

60. Skeath JB. At the nexus between pattern formation and cell-type specification: the generation of individual neuroblast fates in the Drosophila embryonic central nervous system. Bioessays. 1999;21:922-31.

61. Magie CR, Daly M, Martindale MQ. Gastrulation in the cnidarian Nematostella vectensis occurs via invagination not ingression. Dev Biol. 2007;305:483-97.

62. Ormestad M, Martindale $M Q$, Röttinger E. A comparative gene expression database for invertebrates. Evodevo. 2011;2:17

\section{Submit your next manuscript to BioMed Central and we will help you at every step:}

- We accept pre-submission inquiries

- Our selector tool helps you to find the most relevant journal

- We provide round the clock customer support

- Convenient online submission

- Thorough peer review

- Inclusion in PubMed and all major indexing services

- Maximum visibility for your research

Submit your manuscript at www.biomedcentral.com/submit
) Biomed Central 